\title{
Herculanum. Conception du modèle 3D de restitution et restauration virtuelle de la Casa di Nettuno ed Anfitrite
}

Campagne 2016 du projet ANR VESUVIA

Alexandra Dardenay, Hélène Eristov, Adeline Grand-Clément, Marie-Laure Maraval, Nicolas Monteix, Pascal Mora et Maud Mulliez

\section{(2) OpenEdition}

\section{Journals}

Édition électronique

URL : http://journals.openedition.org/cefr/1739

DOI : $10.4000 /$ cefr. 1739

ISSN : 2282-5703

Éditeur

École française de Rome

Référence électronique

Alexandra Dardenay, Hélène Eristov, Adeline Grand-Clément, Marie-Laure Maraval, Nicolas Monteix Pascal Mora et Maud Mulliez, « Herculanum. Conception du modèle 3D de restitution et restauration virtuelle de la Casa di Nettuno ed Anfitrite ", Chronique des activités archéologiques de l'École française de Rome [En ligne], Les cités vésuviennes, mis en ligne le 31 mai 2017, consulté le 30 avril 2019. URL: http://journals.openedition.org/cefr/1739; DOI : 10.4000/cefr.1739

Ce document a été généré automatiquement le 30 avril 2019

(c) École française de Rome 


\title{
Herculanum. Conception du modèle 3D de restitution et restauration
} virtuelle de la Casa di Nettuno ed Anfitrite

\author{
Campagne 2016 du projet ANR VESUVIA \\ Alexandra Dardenay, Hélène Eristov, Adeline Grand-Clément, Marie-Laure \\ Maraval, Nicolas Monteix, Pascal Mora et Maud Mulliez
}

\section{NOTE DE L'AUTEUR}

Notre reconnaissance la plus sincère est adressée aux institutions apportant leur collaboration au projet VESUVIA : Parco Archeologico di Ercolano, Musée Archéologique National de Naples, École française de Rome, Centre Jean Bérard, CNRS, ANR, ENS, Université Toulouse Jean Jaurès, Laboratoire TRACES (UMR 5608)

1 L'un des objectifs du programme ANR VESUVIA (« Vivre Ensemble : Société et Urbanisme d'une Ville de l'Italie Antique») est de reconstituer les différentes phases de l'architecture et de l'ornatus des maisons d'Herculanum. Parmi ses achèvements, le projet prévoit de diffuser une partie de ses méthodes et de ses résultats à travers la réalisation d'un modèle 3D de restitution de la Casa di Nettuno ed Anfitrite d'Herculanum (V, 6-7). Les derniers mois ont été consacrés, d'une part, à la finalisation des infographies de restitution des décors de plusieurs pièces de la maison (les pièces 10, 3, 4 et 7 voir plan, fig.1), et d'autre part, à la conception du modèle 3D par Pascal Mora, d'Archéovision, en concertation avec les membres de l'équipe VESUVIA (fig.2).

2 Si l'on prend soin de respecter certaines règles déontologiques, une restitution 3D peut être un formidable outil heuristique et permet la transmission de résultats issus d'activités archéologiques et historiques. L'interface visuelle que nous avons conçue permet de visualiser plusieurs états de la maison. Les états visibles varient d'une pièce à 
l'autre, en fonction des résultats auxquels nous sommes parvenus. Il nous a également semblé important de pouvoir proposer, le cas échéant, plusieurs hypothèses de restitution, quand les lacunes sont trop nombreuses et que plusieurs versions étaient envisageables.

Fig. 1 - Herculanum, casa di Nettuno ed Anfitrite, plan du rez-de-chaussée, vectorisé par M.-L. Maraval, d'après le plan de Wallace-Hadrill 2011 et Monteix 2010.

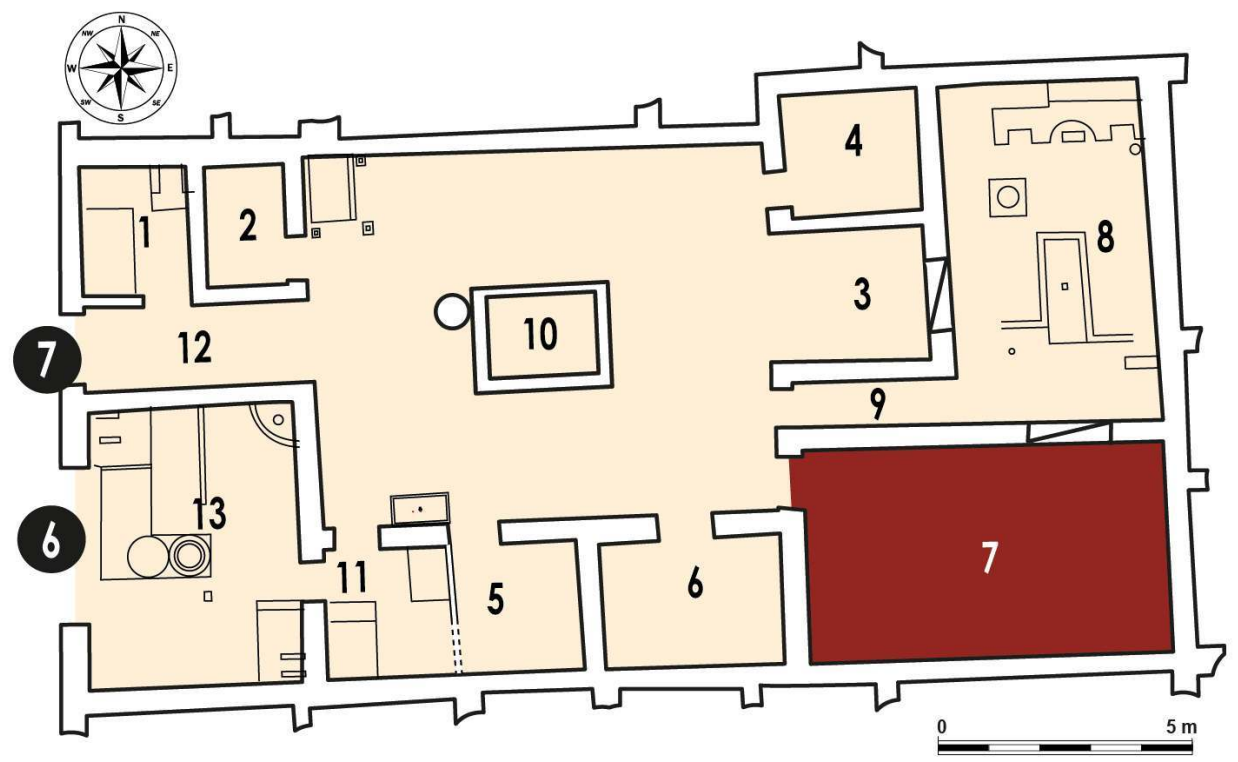

Fig. 2 - Casa di Nettuno ed Anfitrite, Triclinium 7, modèle 3D de restitution, vue vers l'ouest. (c)Archéovision/ANR VESUVIA.

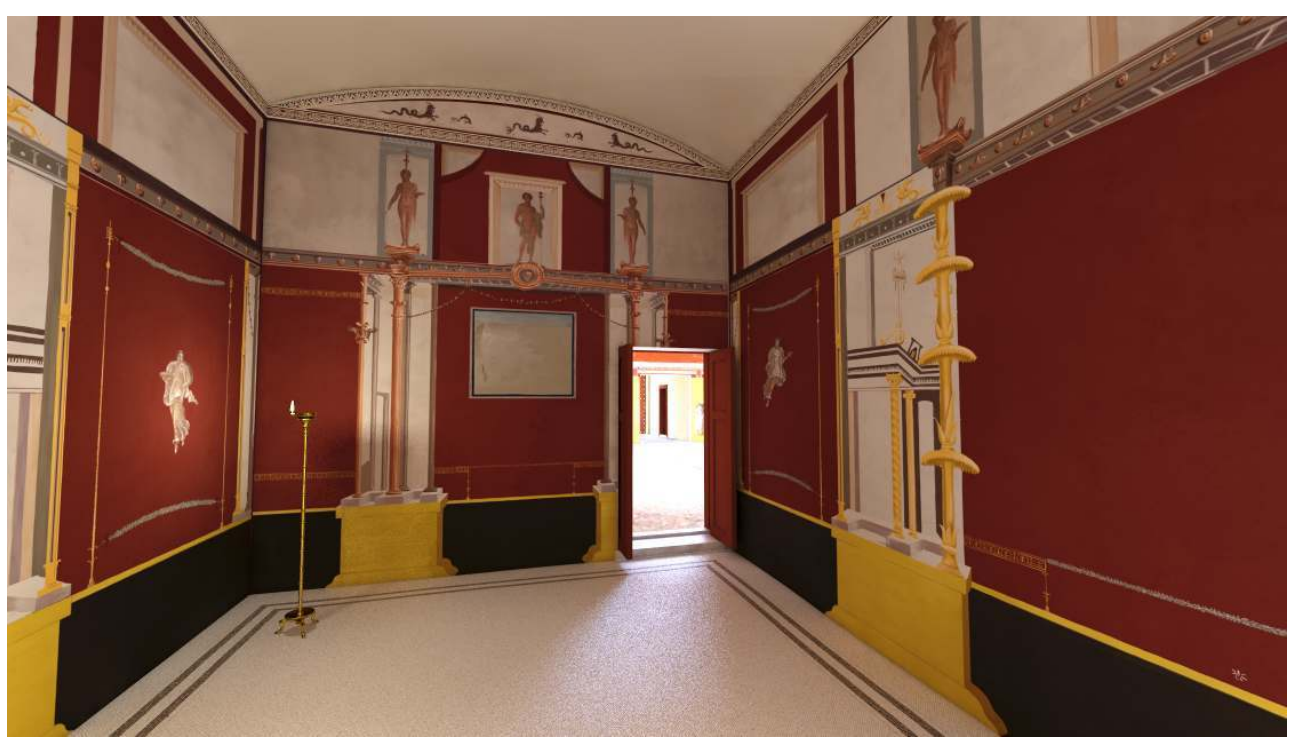




\section{Pourquoi tout restituer?}

3 Pour l'archéologue, la question se pose en premier lieu. Notre formation et nos méthodes de travail habituelles nous encouragent, à juste titre, à limiter l'interprétation aux hypothèses de restitution que nous pouvons fonder, avec un degré de certitude raisonnable, sur les résultats de l'étude du site, de ses vestiges, de ses archives et du matériel qu'il a livré. Mais, comme on le sait, la puissance d'évocation de l'image en fait un des vecteurs de diffusion des savoirs les plus efficaces sur les vestiges de l'Antiquité. Or, il est une fatalité avec laquelle les archéologues doivent composer : avec ou sans eux, notre univers visuel est irrigué d'images de l'antique, d'une antiquité retrouvée, imaginée, fantasmée... Et la demande est toujours plus impérieuse, notamment en raison des progrès spectaculaires réalisés ces dernières décennies, au cinéma et dans les documentaires, dans l'évocation visuelle des villes antiques' ${ }^{1}$. Ainsi, l'image prime, dans le sens où les informations qu'elle véhicule s'imprimeront plus longtemps et plus profondément dans la mémoire que n'importe quel texte, même clair et succinct. C'est pourquoi, en réaction à ce flot d'images incontrôlé et incontrôlable, il apparait du devoir de l'archéologue de proposer des images aussi captivantes, aussi suggestives que possible, mais établies selon des critères scientifiques stricts. Par ailleurs, s'agissant des résultats de campagnes archéologiques, il est important de livrer, en lien direct avec ces images, les documents ayant permis de les réaliser : la mise à disposition des faits, éventuellement ténus, sur lesquels se fondent nos hypothèses est précisément l'un des intérêts de notre démarche. C'est ainsi que les choix opérés seront justifiés.

4 Pour la restitution 3D de la Casa di Nettuno ed Anfitrite, nous avions envisagé dans un premier temps de rendre visible le degré de certitude des éléments restitués par des jeux colorimétriques, ou autres artifices de différentiation. Cette idée a finalement été abandonnée, car nos images, à destination de deux publics - chercheurs et enseignants d'une part, "grand public » de l'autre - aux exigences souvent contradictoires, n'auraient pas su attirer le second groupe autant que d'autres, moins fondées, mais à la puissance de suggestion plus forte parce que répondant à des critères esthétiques désormais intégrés par le plus grand nombre.

5 Afin de concilier exigence scientifique et haute qualité graphique, Archéovision a conçu une interface permettant de faire apparaître progressivement l'état restitué sur l'état actuel, ce qui laisse les restitutions colorées recouvrir puis se substituer aux vestiges. L'affichage des sources et fondements de la restitution est, par ailleurs, mis en œuvre par un système très simple de liens donnant accès soit aux sources de l'élément restitué (archives, photos, gravure...) soit à un bref texte de justification des choix opérés².

\section{Comment rendre perceptibles les différentes étapes d'un travail d'enquête et donc la « valeur ajoutée scientifique »?}

6 Une restitution archéologique comporte nécessairement des manques, des «blancs » qu'aucun élément issu de l'étude scientifique ne permet de combler. Comment limiter la part de décisions arbitraires dans la finalisation intégrale d'un projet 3D? 
7 En schématisant, on peut hiérarchiser notre appréhension de la «certitude » des éléments restitués selon trois degrés.

Le premier degré rassemble les éléments dont la restitution est assurée, car les vestiges sont, depuis leur découverte, dans un état de conservation tout à fait suffisant voire quasi complet. À Herculanum (et dans de nombreux autres sites antiques), les sols, par exemple, entrent dans cette catégorie. Dans le modèle 3D de la Casa di Nettuno ed Anfitrite, Pascal Mora a utilisé pour chaque pièce une ortho-photographie du sol, retravaillée numériquement pour effacer les dommages causés pendant le cataclysme de 79 et depuis la fouille ${ }^{3}$.

9 Le deuxième degré concerne les éléments dégradés, mais à propos desquels l'analyse archéologique, doublée d'une enquête sur les archives de fouilles et les vestiges comparables, a fourni suffisamment d'éléments pour proposer une restitution solidement étayée et argumentée. Toutes nos restitutions de peintures murales entrent dans cette catégorie.

10 Enfin, le troisième degré concerne les éléments détruits, dont les vestiges sont insuffisants pour que l'on ne puisse les restituer sans s'appuyer fortement sur le recours à des raisonnements analogiques. C'est le cas de certains plafonds, de toits ou des portes par exemple, dont les vestiges effondrés ou brûlés n'ont été conservés ou correctement documentés lors de la fouille que dans de trop rares cas. Certains éléments de l'architecture intérieure, surtout dans les étages, entrent également dans cette catégorie.

11 Pour combler les manques, des propositions peuvent être fournies par analogie avec d'autres vestiges plus ou moins contemporains du site étudié ; pour Herculanum, on trouvera des comparaisons utiles à Pompéi, Délos - pour les sites les mieux documentés ou plus sporadiquement, dans d'autres sites d'Italie, de Grèce, de Gaule ${ }^{4}$. Il faut noter que le risque de raisonnement circulaire est ici assez élevé en raison de la forte influence exercée par les sites vésuviens sur les restitutions réalisées dans d'autres sites.

12 Les données issues de l'étude archéologiques sont compilées dans les restitutions 2D, lesquelles font et feront l'objet de publications sur des supports traditionnels. Ces infographies, qui ne sont pas principalement destinées au grand public mais à la communauté scientifique, s'attachent avant tout à mettre en évidence les résultats de l'analyse et ne présentent que les éléments dont la restitution est assurée ou les différentes hypothèses que nous pouvons proposer (soit les premier et deuxième degrés de la hiérarchisation précédemment évoquée). Tous les éléments restitués selon de simples critères de vraisemblance dans le modèle 3D sont alors éliminés.

\section{Le cas de l'atrium et du laraire de la Casa di Nettuno ed Anfitrite}

13 Nous avons présenté dans une précédente Chronique les éléments et arguments sur lesquels étaient appuyées nos restitutions 2D du décor pariétal ; nous aborderons ici des questions plus strictement architecturales (fig. 3).

Le pavement est un béton de tuileau gris rosé, de granulométrie assez grossière avec des inclusions de pierres et de grands éclats de marbre irréguliers de diverses couleurs. Dans la monographie parue récemment sur les sols d'Herculanum ${ }^{5}$, il est daté stylistiquement du IV style, et interprété comme de réfection contemporaine au dernier état du décor 
des parois, soit dans les années 70 d'après notre étude. Sa facture est plutôt grossière et l'exécution manque de raffinement. Néanmoins, sa restitution ne pose pas de problème particulier.

Fig. 3 - Casa di Nettuno ed Anfitrite, Atrium, modèle 3D de restitution, vue vers l'ouest.

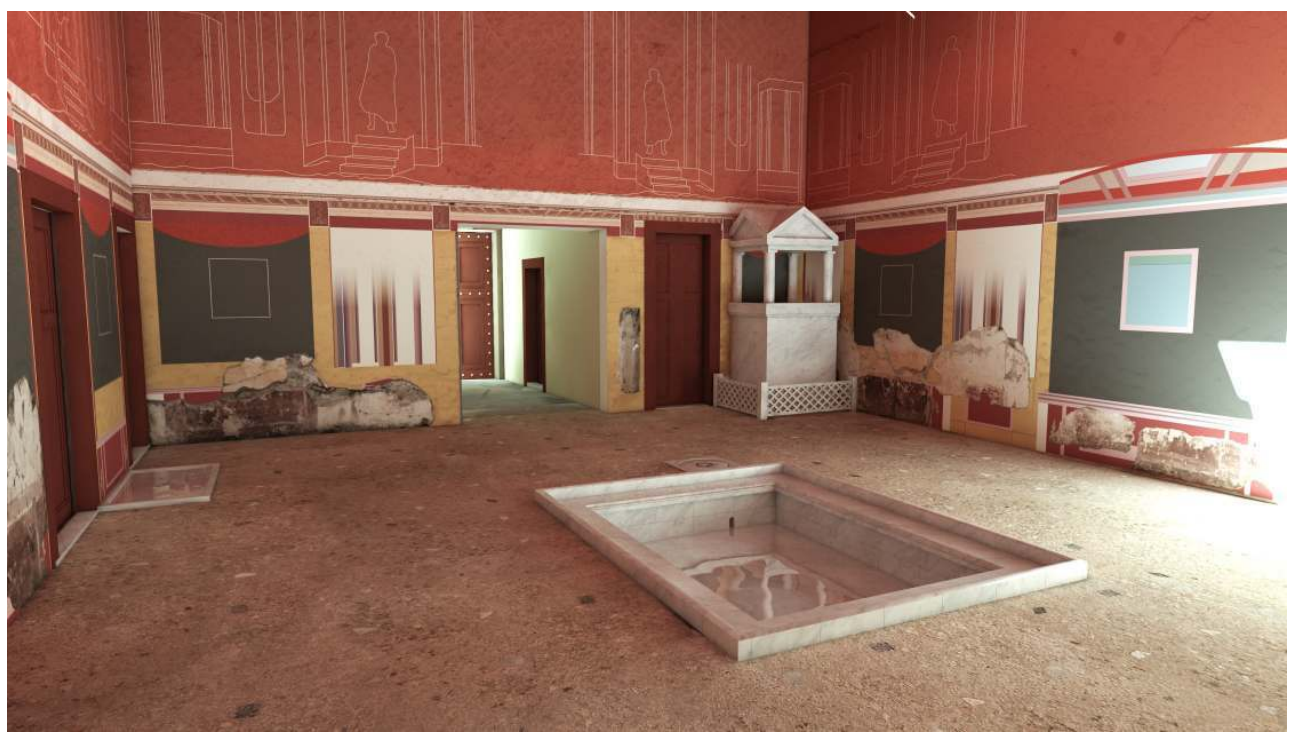

Version non définitive (travail en cours).

(c) Archéovision/ANR VESUVIA.

\section{La couverture de l'atrium (fig. 4)}

En dépit de la bonne conservation des élévations dans la Casa di Nettuno ed Anfitrite, la restitution de la couverture de l'atrium soulève des questions qui peuvent, ou non, être résolues. La forme générale est celle d'un toit en tronc de pyramide renversée dont la partie centrale est laissée découverte. Les dimensions de ce vide nous sont données par celles du bassin recevant les eaux de pluie, directement ou après ruissellement sur la toiture. De forme rectangulaire, l'impluvium mesure 2,02 m de longueur pour $1,50 \mathrm{~m}$ de largeur. Bien que son emprise déborde de $0,25 \mathrm{~m}$ de chaque côté à cause de la présence d'un rebord, il nous est apparu plus raisonnable de considérer les dimensions du bassin stricto sensu comme identiques à celles du vide dans la toiture : le rebord servant alors de protection contre les déplacements de l'eau lors de sa chute. La charpente primaire est constituée de deux poutres transversales dont les dimensions sont connues grâce à l'empreinte de leur section dans le mur septentrional ${ }^{6}$. La bonne conservation des murs occidental et septentrional permet également de connaître les dimensions et l'espacement des chevrons ${ }^{7}$. Nous ne disposons d'aucune trace spécifique qui permettrait de caractériser les chevrons de noue qui constituent les axes d'interpénétration des pans de toiture deux à deux. Il semble cependant indispensable de les restituer dans l'angle : le sommet de tous les chevrons se trouve à la même altitude ${ }^{8}$. Un point reste - et restera l'objet de conjectures : la nature de la poutre à laquelle les chevrons sont fixés dans leur partie inférieure ainsi que la forme prise par cet assemblage. Pour simplifier, nous avons considéré que l'ensemble des chevrons s'achevait sur un quadrilatère de pièces de bois, reposant sur les deux poutres transversales de la charpente primaire. 
Les empreintes des tuiles - plates et couvre-joints - sont conservées dans le seul mur occidental. Il est important de noter que le rythme des couvre-joints est différent de celui des chevrons, ce qui impose de restituer un platelage, constitué de voliges, ou, eu égard au faible espace entre le sommet des chevrons et la base des tuiles, un lattis à même de recevoir les tuiles. Nous avons restitué des tuiles de noue, en suivant un modèle attesté sur le site. Des couvre-joints d'égout à antéfixes à tête de chien présentant des traces de peinture blanche ont également été restitués suite à leur découverte lors de la fouille de l' atrium 9 .

Fig. 4 - Casa di Nettuno ed Anfitrite, Atrium, détail du compluvium. (c) Archéovision/ANR VESUVIA.

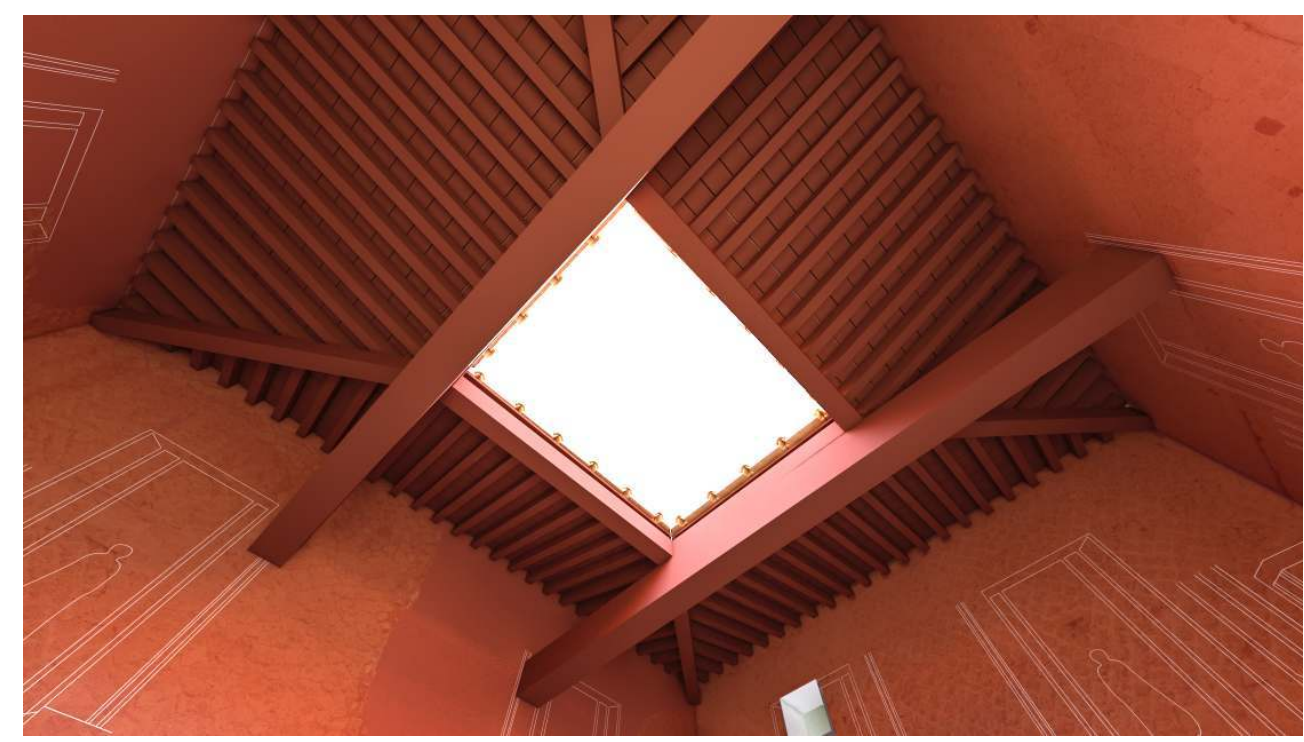

\section{L'ornatus de l'atrium}

Outre le mobilier de bois de la pièce, dont aucun élément n'a été identifié lors des fouilles, l'ornatus de l'atrium comportait un imposant laraire maçonné découvert dans un état très altéré lors des fouilles d'A. Maiuri en 1933. Concernant ce regrettable état de conservation, l'hypothèse la plus probable est que ce laraire, appuyé contre le mur nord, aurait été fortement endommagé par les excavations commissionnées par les Bourbons au XVIII siècle. L'enquête menée par Agnes Allroggen-Bedel a révélé que les fouilleurs se trouvaient dans cette maison au printemps $1746^{10}$ et qu'au moins deux cunicoli traversaient la maison, dont l'un transperçant le mur nord à l'emplacement du laraire : dans les journaux de fouille (GSE), à la date du 19 avril 1933, est mentionnée la découverte du massif du laraire, dont la partie haute aurait été détruite par le passage d'un tunnel sous les Bourbons ${ }^{11}$. À la même date, est signalée la découverte, tout près de ce massif, de deux statuettes de bronze, l'une figurant un chameau $\left(\mathrm{n}^{\circ} 1059\right)$ et l'autre un petit hermès de bronze $\left(\mathrm{n}^{\mathrm{o}} 1060\right)^{12}$. Il est possible que ces deux statuettes aient fait partie de l' instrumentum du laraire.

Le terme d'« évocation » est celui qui paraît le plus approprié pour la reconstruction du laraire sur laquelle l'équipe du programme VESUVIA travaille actuellement. En effet, peu de vestiges en sont conservés, à part le massif in situ, et nous n'avons aucun élément provenant de l'architecture de la partie haute du petit monument. Après consultation des 
corpus de laraires campaniens publiés ${ }^{13}$, nous avons opté pour une architecture assez générique : l'épaisseur et la largeur du soubassement sont données par les vestiges de maçonnerie in situ ; quant à la hauteur, on suppose que le plan de l'autel devait se trouver à environ 1,20 ou $1,30 \mathrm{~m}$ du sol, pour permettre à un homme debout de réaliser ses rituels. En couronnement, nous envisageons un couvrement à fronton supporté par quatre colonnettes, dont les exemples abondent dans les laraires maçonnés campaniens ${ }^{14}$. Étant donné que le laraire n'est pas collé contre le mur nord, la partie au-dessus de la base aura une composition symétrique, comme s'il était au milieu d'une pièce. Enfin, l'autel pourrait avoir été fermé par de petits volets de bois, comme celui de la casa del Menandro à Pompéi (I 10, 4) $)^{15}$.

Concernant le décor du laraire, les GSE signalent, à la date du 20 avril 1933, la présence de nombreux fragments de marbres qui jonchaient le sol dans l'angle nord-ouest de l'atrium, lesquels sont attribués d'emblée à l'ornement de l'autel domestique. Nous avons retenu l'hypothèse, la base du massif présentant elle-même des éléments de placages de marbre. C'est ainsi que l'évocation du laraire le représente entièrement recouvert de marbre, bien que les parallèles soient rares $^{16}$ et qu'il offre ainsi un luxe un peu extravagant, surtout quand on considère la grossièreté du pavement. Mais nous avons pu montrer que le décor peint de l'atrium était en cours de réfection ${ }^{17}$, ainsi, peut-être qu'un laraire de marbre tout neuf, lui aussi, venait afficher un regain de fortune du dominus.

Les deux pinakes de marbre découverts dans l'atrium en 1933, déjà évoqués assez longuement dans une précédente Chronique, constituent eux aussi un élément de décor luxueux de cet espace. Dans les GSE ils sont signalés à la date du 20 avril, comme ayant été découverts à proximité de l'autel et Maiuri, dans son ouvrage de 1958, proposait de les restituer comme ornement du laraire ${ }^{18}$. La proposition s'est imposée depuis lors, et ces deux plaques de marbres figurées sont toujours mentionnées dans les publications comme des vestiges du décor du laraire de la casa di Nettuno ed Anfitrite. Le problème est que cela constituerait alors un hapax, jamais des tableaux peints sur marbre n'ayant été attestés comme décor de laraire. Il existe de nombreux exemples de décors figurés dans le cas des autels domestiques, mais ils sont alors peints sur le mortier du mur du fond, ou de part et d'autre ${ }^{19}$. Il est vrai, toutefois, que les découvertes de pinakes de marbre sont très rares et donc leur manifestation dans l'ornatus de la maison romaine reste très mal connue à ce jour. En revanche, ils se rapprochent beaucoup, matériellement, du corpus des petits reliefs de marbre néo-attiques, très à la mode au début de l'époque impériale, et qui s'inséraient dans les murs peints des décors de $\mathrm{II}^{\mathrm{e}}$ style tardif, de $\mathrm{III}^{\mathrm{e}}$ ou de $\mathrm{IV}^{\mathrm{e}}$ styles pompéiens ${ }^{20}$. Pour ce type de décor figuré, rapporté dans une paroi, les exemples abondent en revanche, aussi bien dans la littérature ancienne ${ }^{21}$ que dans les découvertes archéologiques qui permettent de comprendre comment ces petits reliefs de marbre étaient fixés dans le mur ${ }^{22}$.

Dès lors, faute de parallèle archéologique et de réponse satisfaisante à la question de savoir où les deux pinakes de marbre de l'atrium de la Casa di Nettuno ed Anfitrite - qui mesuraient entre 40 et $50 \mathrm{~cm}$ de côté ${ }^{23}$ - pourraient être restitués dans l'architecture du laraire, nous avons envisagé une autre hypothèse. En s'appuyant sur le parallèle avec les reliefs néo-attiques, il serait envisageable de les replacer dans les parois de l'atrium, dont le décor peint présentait une scénographie de $\mathrm{IV}^{\mathrm{e}}$ style. La restitution infographique que nous avons proposée du décor montre l'emplacement de tableaux dont on suppose l'existence, mais qui n'ont pas été conservés, à l'exception d'un tableau à sujet mythologique peint sur la paroi ouest, dont A. Maiuri signale la présence, mais qui s'est 
effacé depuis lors ${ }^{24}$. Par ailleurs, notre enquête dans les réserves du Musée Archéologique National de Naples (MANN) n'a pas livré jusqu'ici de tableaux prélevés au XVIII ${ }^{\mathrm{e}}$ siècle qui puisse être restitués dans ce décor. On sait que le décor pariétal de l'atrium était en cours de réfection, notamment grâce à la découverte, sur la paroi ouest, d'une inscription purpura qui indiquait la couleur à appliquer sur une bordure ${ }^{25}$. On peut donc supposer que les tableaux n'étaient pas encore tous peints, puisque c'était une partie du décor que les ateliers de peintres ne réalisaient généralement qu'à la fin du chantier ${ }^{26}$.

Un autre problème de restitution est posé par les trois trous d'ancrage présents dans le sol à trois des angles du laraire. Ces quadrilatères de marbre blanc évidés en leur centre sont insérés dans le béton formant le sol de l'atrium. De quel aménagement pourraient-ils être la trace? Nous avons envisagé, pour le moment, plusieurs solutions : soit une petite barrière à claustra (ou un pluteus) maintenue par trois montants fichés dans ces trous d'ancrage, soit des piliers simples ou couronnés de figures hermaïques. Une troisième hypothèse y verrait la trace d'un aménagement antérieur au dernier état et dont nous ignorons tout.

On le constate, pour ce qui est de la restitution de l'ornatus de l'atrium, nous sommes confrontés à un exemple typique d'emboitements d'hypothèses suscitées par les manques et les doutes résiduels, qui encouragent à multiplier les propositions. Plusieurs états de restitutions pourraient et devraient être proposés. Concernant les fameux pinakes, dans la mesure où ils n'étaient pas ou plus, au moment de leur découverte à l'endroit qui leur était destinés, nous envisageons tout simplement de les placer au sol, contre une paroi, près de l'endroit où ils ont été découverts.

\section{Mise à jour des infographies du décor de l'atrium}

Si le décor des zones basse et médiane de l'atrium avait pu être restitué en fonction des vestiges in situ, rien ne subsiste aujourd'hui de la zone supérieure, haute de $3 \mathrm{~m}$, située en-dessous des solives. Seul un cliché de 1935 publié par A. Maiuri ${ }^{27}$ témoigne de trois lambeaux d'enduit sur le mur est, l'un sur le tiers gauche, l'autre sur le tiers droit un dernier, plus petit, à l'angle droit de la paroi. Tous ont totalement disparu aujourd'hui à l'exception du bandeau correspondant à l'entablement de la zone médiane au-dessus des portes menant au couloir [9] et au triclinium [7]. Le cliché en noir et blanc ne nous renseigne que sur les valeurs claires et sombres mais la bonne qualité du tirage original a fourni des informations précieuses (fig. 5). 
Fig. 5 - Casa di Nettuno ed Anfitrite, Atrium, à gauche cliché d'archive : état en 1935 (photo Alinari n • 43124 / Roger Viollet 99453-3) et détail retravaillé ; à droite, état actuel et détail.
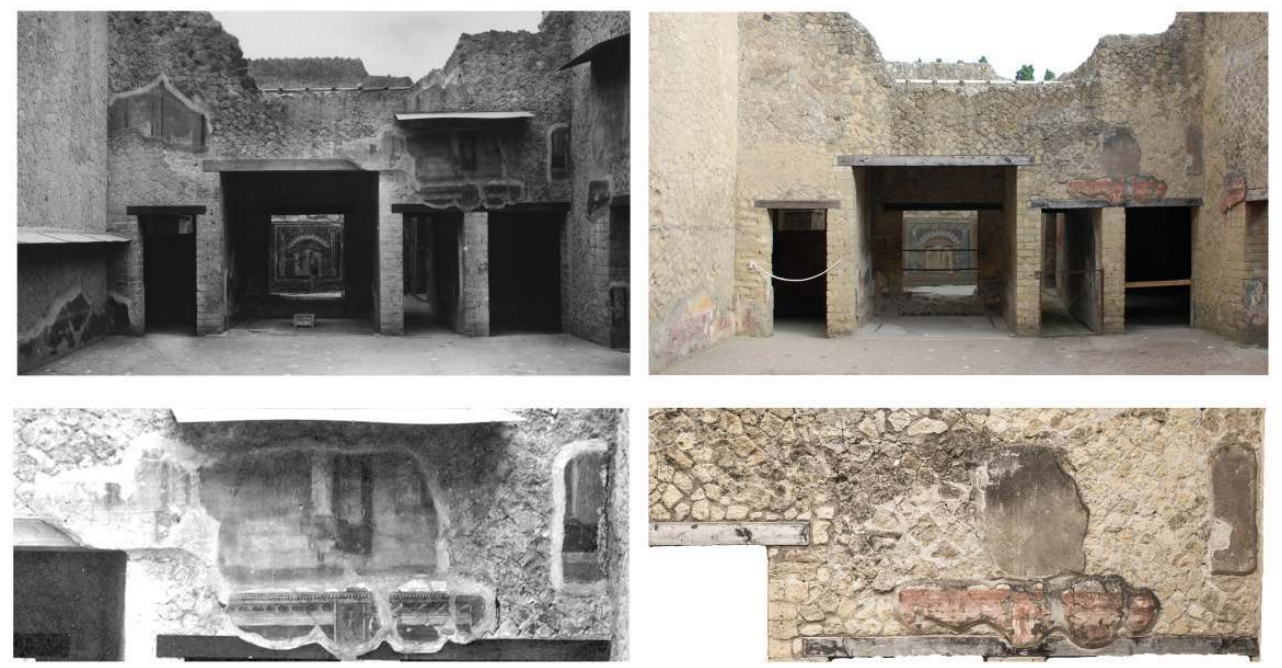

Le fragment conservé d'entablement de la zone médiane, aujourd'hui très délavé, est beaucoup plus lisible sur la photo d'archive : il témoigne de l'alternance, au-dessus des champs médians, d'un soffite à caissons quadrangulaires et d'une corniche à consoles en forme de X, la transition étant assurée par un petit compartiment rectangulaire occupé par une harpie encore partiellement visible. Au-dessus, court une modénature continue en forme de perles. Il est à noter qu'entre la photo d'archive et l'état actuel, les fragments d'entablement « in situ » ont bougé : la figure hybride, aujourd'hui centrée sur le montant entre les deux portes, se situait nettement plus à gauche en 1935, ce qui pose une fois de plus la question de la fiabilité des repositionnements.

Le fragment du tiers droit, le plus important, laisse clairement voir les degrés d'un escalier vu en perspective latérale, flanquée à droite par un distyle fuyant à muret, et à gauche par l'amorce d'un pan frontal. Au-dessus de l'escalier subsistent les traces de deux pieds et d'un vêtement incurvé qui signalent la présence d'une figure. Il s'agit donc du dispositif décoratif déjà rencontré dans le triclinium [7] et dans la pièce [4], caractérisé par des échappées architecturales à pavillons en vue latérale précédés par des escaliers en haut desquels se tiennent des figures.

La répétition symétrique de ce fragment sur le tiers gauche amène à compléter la composition: des séquences alternativement claires et sombres suggèrent un enchaînement architectural, tandis qu'une forme concave se laisse interpréter comme la base d'un possible trépied, ou du moins d'une structure triangulaire. Aux angles, le lambeau conservé du côté sud laisse entrevoir des supports et/ou des cloisons sur un haut piédestal. La photo d'archive montre un écart dimensionnel entre la gauche et la droite du mur est : le pavillon à l'extrémité gauche est large de $0,79 \mathrm{~m}$, celui de droite de 0,65 m. Seul le centre de la composition (soit une largeur de 1,20 m) nous reste inconnu. La hauteur de l'ensemble, hypothétique, a été restituée sur 2,25 m, en fonction de la hauteur probable des figures; en revanche le couronnement, qui n'a laissé aucune trace, a été laissé vide dans la restitution (soit une hauteur résiduelle de 0,75 m) (fig. 6). 
Fig. 6 - Restitution du décor de l'atrium (mur est) (DAO Vesuvia/M.-L. Maraval, DAR (Direction en appui à la recherche), université Toulouse Jean-Jaurès).

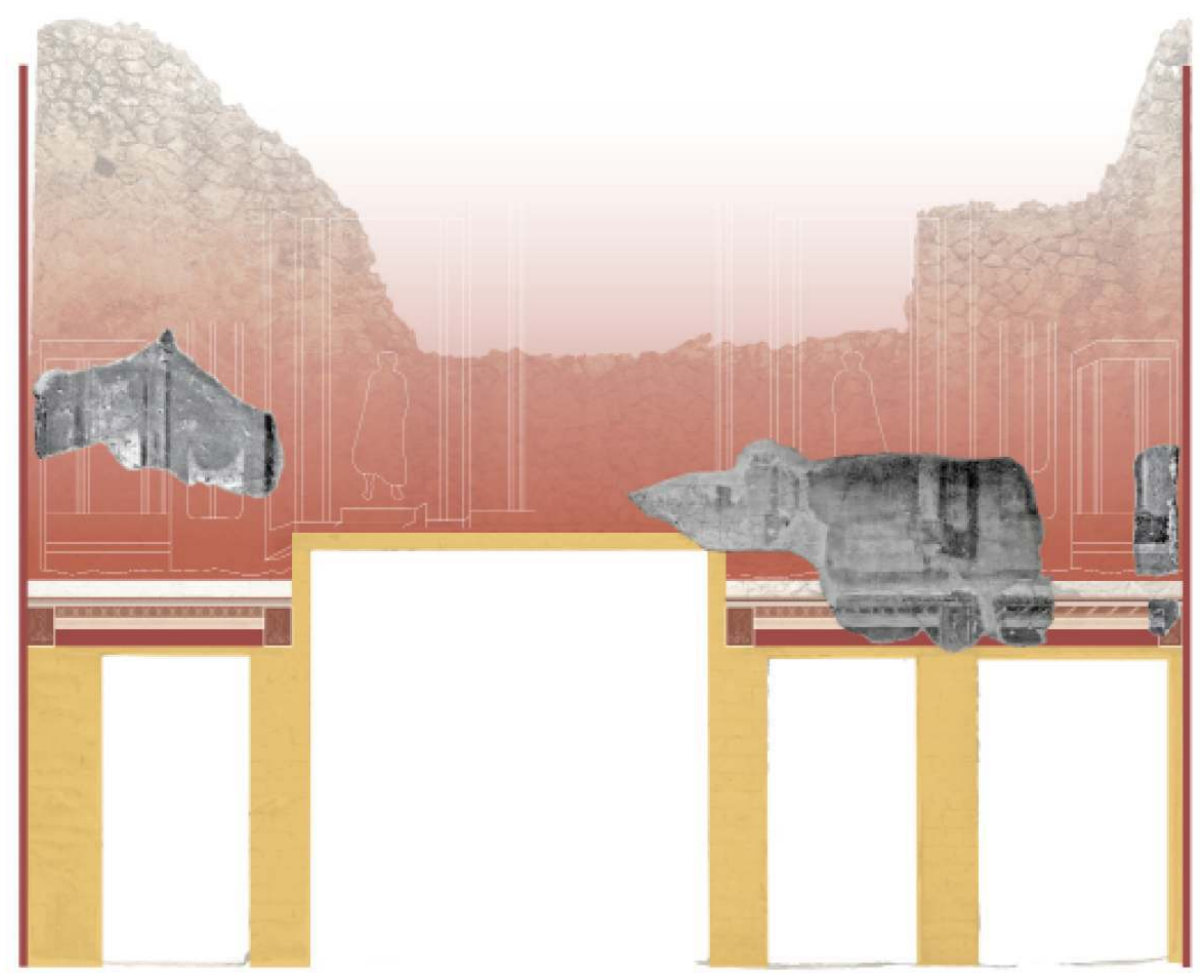

Seul le mur oriental a été documenté à l'époque des fouilles, alors que la description d'archives comme celle d'A. Maiuri ${ }^{28}$ signalent le mur occidental comme le mieux conservé et que, de toute évidence, un important lambeau du mur méridional était conservé, comme en témoigne la petite avancée de toiture installée lors des fouilles pour le protéger, en vain, malheureusement. Cependant, dans la mesure où les choix décoratifs adoptés dans la maison privilégiaient une répétition quasi à l'identique des quatre murs d'une même pièce, il a été décidé de répéter le même dispositif de zone haute sur tous les murs de l'atrium, sachant que les proportions différaient sensiblement et que les motifs architecturaux pouvaient être étirés. Enfin, dans l'ignorance où nous sommes des couleurs, le parti-pris de restitution retenu a consisté à traiter les motifs architecturaux et figurés de manière linéaire sur un fond rouge: les valeurs de gris constatées sur le cliché dénotent en effet un fond soutenu et non une composition architecturale sur fond blanc.

La grande homogénéité du décor de la maison, de toute évidence réalisé en une seule phase et par le même atelier, se vérifie donc dans l'atrium.

\section{Restitution du décor du tablinum [8]}

Il subsiste du mur sud la quasi-totalité du soubassement et le bas du champ central ; le mur nord très érodé a conservé son soubassement, le champ central jusqu'à mi-hauteur, le tiers inférieur de l'échappée gauche et le bas de l'échappée droite. Les décors in situ sont complétés par un fragment aujourd'hui conservé au musée du Louvre (P14) ${ }^{29}$ mais 
primitivement au musée de Portici sous le numéro CLVII ${ }^{30}$; il est documenté vers 1804 par un dessin d' Élie-Honoré Montagny ${ }^{31}$ (fig. 7).

Fig. 7 - Elie-Honoré Montagny, Recueil d'antiquités, folio 32 (The Getty Research Institute, Los Angeles).

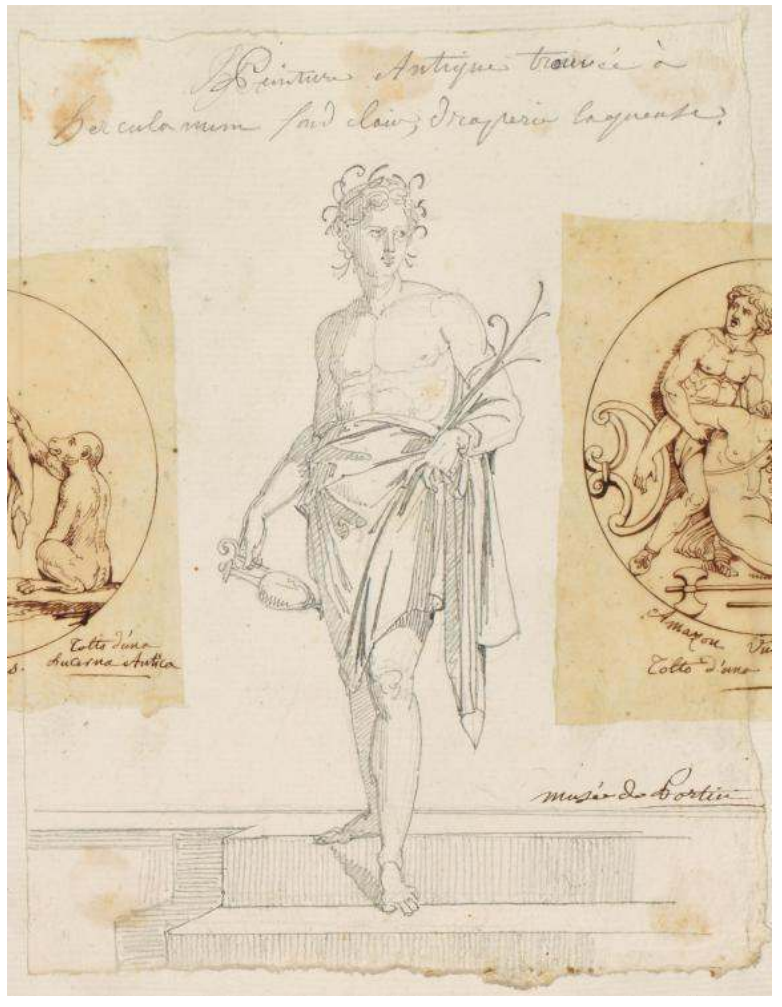

Comme en d'autres occurrences, le sol présente une forte inclinaison due au compactage différencié des remblais sous-jacents après l'éruption, de sorte que l'extrémité est de la pièce se trouve $13 \mathrm{~cm}$ plus bas que l'extrémité ouest. Il a donc été pris le parti de redresser l'ensemble afin d'installer la ligne de soubassement à l'horizontale.

Le schéma d'ensemble est clairement lisible : le soubassement rouge-bordeaux est articulé par deux gros piédestaux ocre jaune qui séparent les étroits panneaux latéraux, uniquement occupés par un encadrement linéaire en-dessous d'un plafond à solives, de part et d'autre du large panneau central orné d'un candélabre bas à volutes traversé par une bordure ajourée horizontale, l'ensemble inscrit dans une baie fictive. En zone médiane, un édicule central dont le couronnement est perdu se présentait sous la forme d'une tenture incurvée bleue bordée d'une guirlande et servant de fond à un tableau fragmentaire. De part et d'autre, des échappées architecturales comportent un escalier que s'apprête à descendre une figure.

Le détail des représentations pose, en revanche, quelques difficultés de lecture notamment dans la construction architecturale de l'échappée. L'escalier s'appuie sur un haut piédestal orné de feuilles, absolument similaire à ceux de la pièce [4] ; mais le rendu des ressauts et des ombres portées a visiblement dépassé les capacités du peintre et induit une erreur d'interprétation chez les modernes : à la différence d'É.-H. Montagny, le RPGR de S. Reinach traduit les ombres comme une bordure de tapis d'escalier, ce qui sera repris par les restaurateurs du Louvre. La lecture des clichés des années 1970 et du fragment du Louvre, ajoutée aux comparaisons avec des dispositifs semblables permettent de 
restituer, au-dessus de l'escalier, un pavillon porté, vers l'extrémité de la paroi, par une colonne cannelée sur un ressaut de piédestal. La restitution de la moitié haute de l'échappée s'appuie sur la succession du fût cannelé et de pans clairs et sombres déterminant un mur fuyant et un retour frontal dans lequel s'ouvre une porte, un vantail ouvert, l'autre fermé.

Par analogie avec l'échappée de la pièce [4], il est vraisemblable qu'ici aussi deux niveaux architecturaux se superposaient, le second très simplifié. La reprise de ces proportions permet de proposer une hauteur de $0,80 \mathrm{~m}$ pour le premier niveau (fig. 8).

Fig. 8 - Restitution du décor du tablinum ((DAO Vesuvia/M.-L. Maraval, DAR (Direction en appui à la recherche), Université Toulouse Jean-Jaurès).

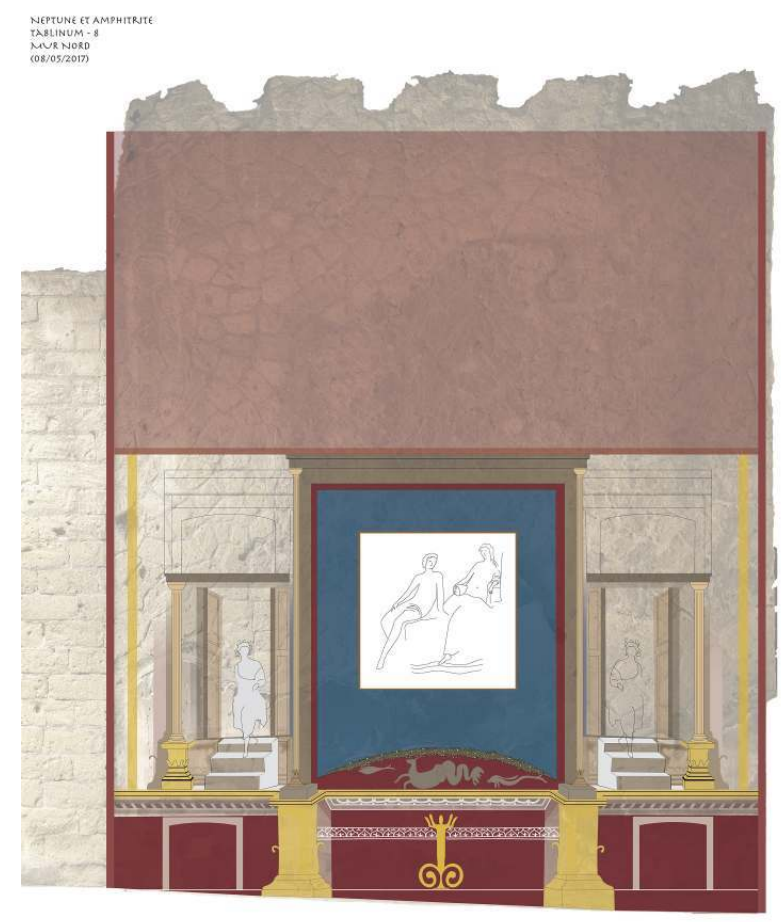

Vers le centre de la paroi, une colonne cannelée à base végétalisée sur un dé porté par le haut piédestal jaune du soubassement se situe au premier plan, nettement en avant de l'escalier et du pavillon; elle est donc à identifier comme l'un des supports de l'édicule central de la composition. En l'absence de toute donnée la restitution propose un entablement plat.

Il subsiste in situ le bas du corps de la figure de l'échappée gauche du mur nord (fig.9) et sur le fragment du Louvre celle de l'échappée gauche du mur sud (fig.10). Tous deux sont vêtus d'un manteau court qui laisse les jambes nues et, sur le fragment du Louvre, également le torse. Visiblement ces deux figures étaient très semblables, celle du Louvre tenant une œnochoé ou un lécythe, et une palme au creux du bras gauche, est couronnée de tiges végétales qui lui confèrent un caractère dionysiaque. Le traitement semble-t-il effiloché du manteau de la figure in situ pourrait correspondre à une nébride. 
Fig. 9 - Tablinum, mur nord : personnage dans l'échappée gauche (cliché M.-L. Maraval).

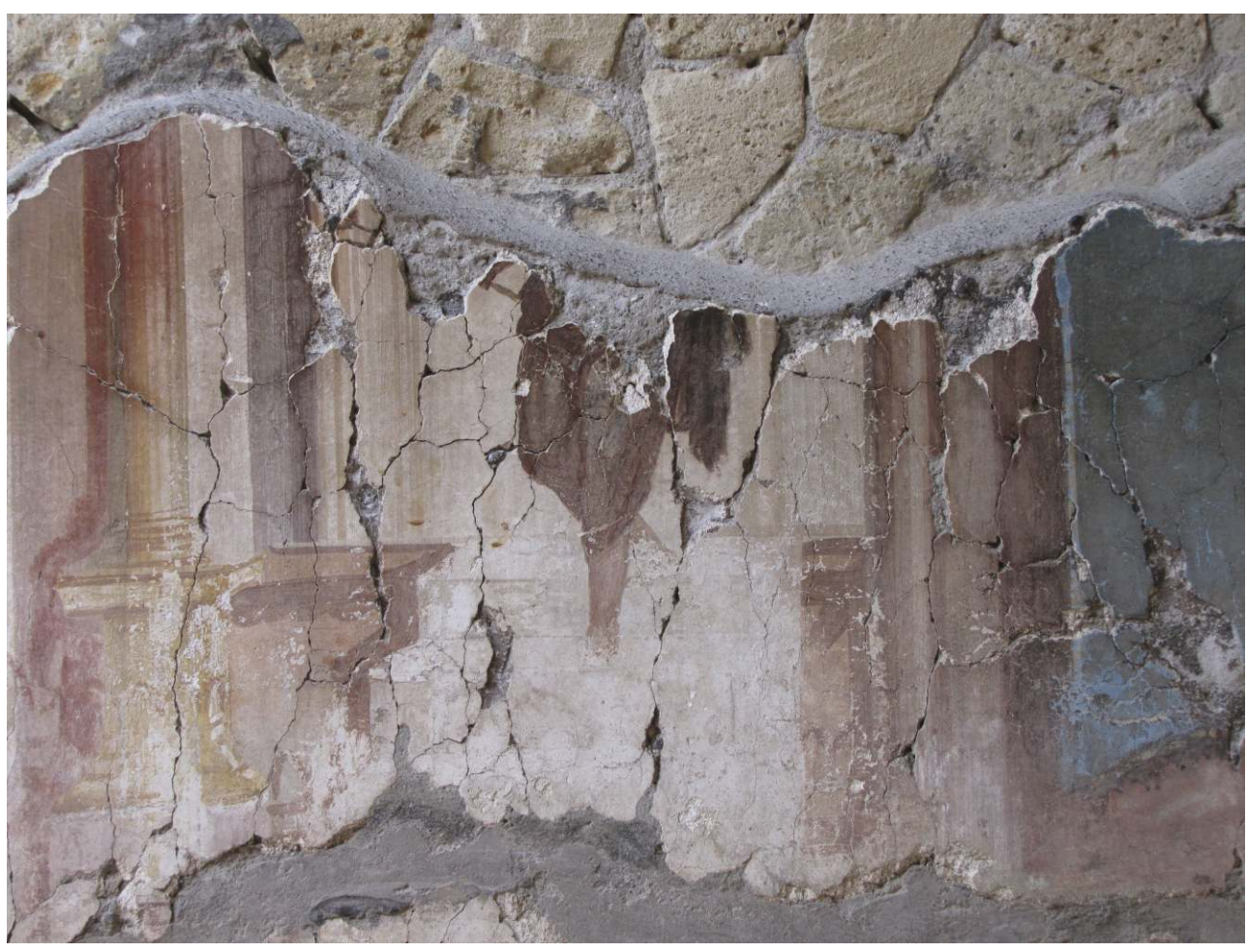

Fig. 10 - Panneau conservé au Louvre (inv. P 14) : personnage dans l'échappée gauche du mur sud (photo D. Burlot).

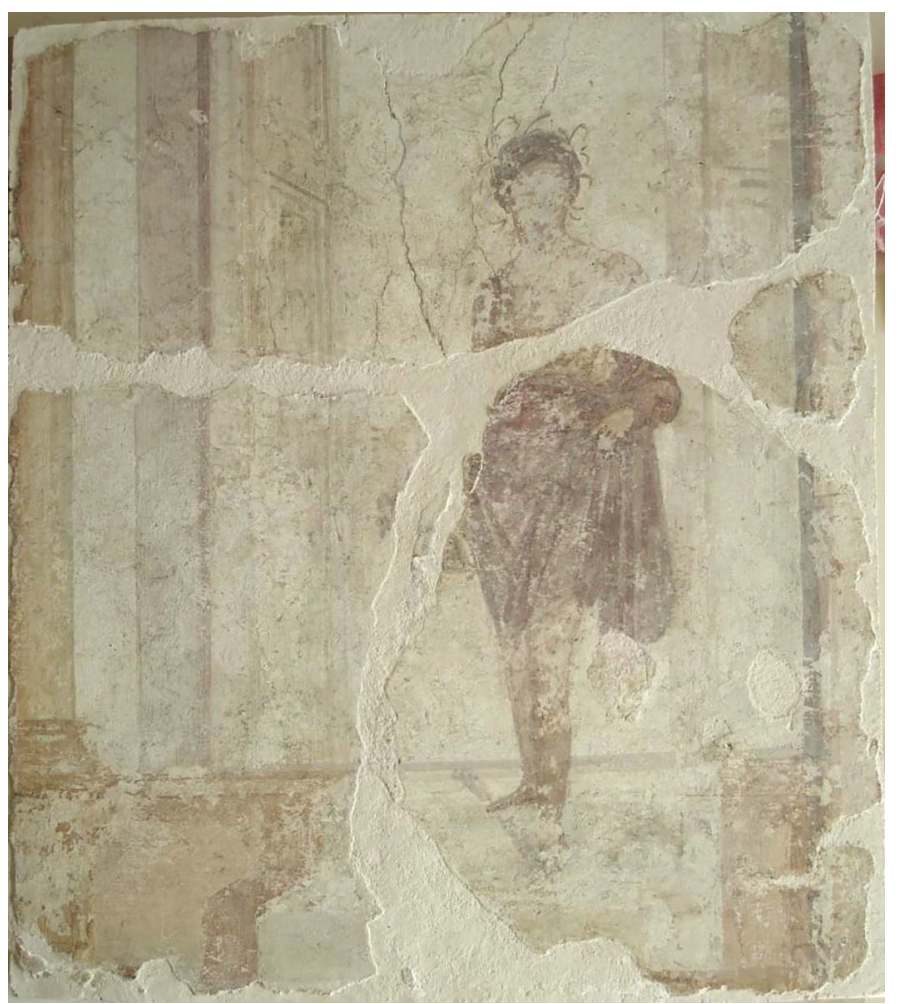

37 Au centre de la paroi, un tableau très endommagé (dimensions restituées : 0,62 m de côté) encadré de deux filets laisse encore discerner le bas du corps de deux figures identifiables 
comme Narcisse et une figure féminine assise un peu plus haut derrière lui, la main droite posée sur une urne et la gauche maintenant une tige de roseau dans l'attitude habituelle des nymphes de sources. La présence d'un Amour reste incertaine ici, mais quoi qu'il en soit la formule iconographique est inhabituelle: la plus proche ${ }^{32}$, celle, aujourd'hui disparue, de la pièce [38] de la Casa dei Dioscuri (VI, 9, 6-7), comme aussi celle de la Casa dell'argenteria (VI 7,20; MANN inv. $\left.n^{\circ} 9388\right)^{33}$, montrent la nymphe des sources assise à l'arrière-plan (fig.11), tandis qu'ici, de même importance que le héros et sur le même plan que lui, elle réplique son attitude. Il est à noter que ce thème élégiaque ne trouve habituellement pas sa place dans un tablinum à l'exception de la Casa dell'argenteria à Pompéi ${ }^{34}$ où il est associé à Endymion. La position de Narcisse inversée par rapport à celui d'Herculanum, se justifie sans doute par le fait qu'il se trouve sur le mur droit du tablinum, de sorte que, comme à Herculanum, il fait face à l'entrée de la pièce.

Fig. 11 - Narcisse-Helbig 1364, d'après RMB I.4.

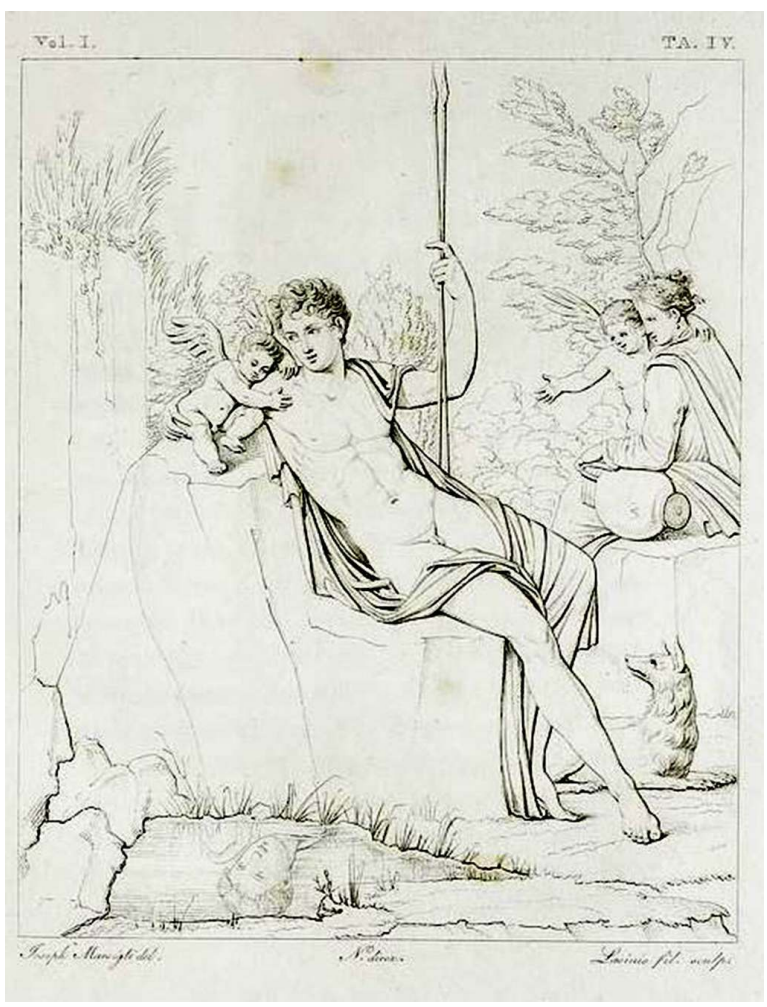

En-dessous de l'incurvation de la tenture bleue centrale, sur les murs nord et sud, un compartiment à fond rouge porte un ichtyocentaure encadré de dauphins. La répétition identique du motif sur les deux murs confirme le jugement général déjà porté sur les décors de cette maison où les décorateurs se sont contentés de reproduire les mêmes formules.

\section{Réalisation du modèle numérique}

Le modèle numérique 3D utilisé pour ce travail a été élaboré en plusieurs étapes. Tout d'abord, une numérisation tridimensionnelle de la Casa di Nettuno ed Anfitrite par photogrammétrie par corrélation dense a été faite sur le terrain en 2014. Durant cette phase préliminaire du travail, un grand nombre de prises de vues de haute qualité de 
l'ensemble des pièces de la maison ont été réalisées, ces photographies devant pouvoir être utilisables pour le calcul photogrammétrique mais aussi pour la production d'orthoimages. Environ 1500 clichés en 36 mégapixels ont été nécessaires pour obtenir un modèle numérique 3D très complet de l'ensemble de la maison avec une résolution infracentimétrique. C'est le logiciel de photogrammétrie Photoscan (Agisoft) qui a été utilisé pour cette phase du travail, le modèle 3D obtenu a ensuite été géo-référencé à l'aide de points topographiques relevés au tachéomètre par Guilhem Chapelin, architecte du Centre Jean Bérard.

40 Les données 3D obtenues ont ainsi pu être mises à l'échelle, nettoyées, optimisées et maillées afin d'avoir un modèle 3D surfacique exploitable pour la restitution de l'architecture et la production d'ortho-images.

41 Cependant, la géométrie issue de la numérisation 3D étant trop complexe, une remodélisation plus simple de l'ensemble de la maison dans un logiciel de modélisation 3D généraliste (3Ds max) a été nécessaire afin de poursuivre les opérations de restitution.

42 La bonne conservation des vestiges a permis, la plupart du temps, d'utiliser directement le modèle numérique 3D pour caler précisément bon nombre d'éléments architecturaux de la maison: poutraison du compluvium, positionnement du laraire, du bassin de l' impluvium, positionnement des pivots des portes, etc. Certains éléments de mobilier, entreposés dans les réserves du site, ont été également numérisés par photogrammétrie et, après avoir également été re-modélisés, ont pu être intégrés dans la restitution de certaines pièces de la maison.

Le modèle numérique et les ortho-images ont été d'une grande aide pour dimensionner et positionner les murs avec précision et permettre un placement précis des peintures restituées. Les ortho-images des murs peints ont servi de calques précis pour recaler les restitutions et faire gagner un temps précieux en épargnant de nombreuses mesures sur le terrain.

Des panoramas $360^{\circ}$ ont pu être réalisés sur le terrain en utilisant une tête panoramique adaptée au milieu de chaque pièce de la Casa di Nettuno ed Anfitrite. Exactement au même endroit dans le modèle 3D restitué, un même panorama a été calculé dans plusieurs versions du modèle 3D (restitution de l'architecture seule ou de l'architecture et des décors). Les points de vue étant identiques, les images obtenues sont parfaitement superposables et autorisent donc la réalisation de fondus entre les différents états. Les différentes phases du travail de ce projet sont consultables et exploitables de façon interactive grâce à un simple navigateur Internet. Une interface permet de naviguer virtuellement dans la maison et de visualiser les différentes étapes du travail de recherche, de la restitution du décor et de l'architecture jusqu'à la restauration numérique fine des peintures.

\section{Restauration numérique des peintures murales}

Grâce à un croisement avec le programme Retro-Color3D porté par Archéovision, la restitution "totale" des peintures murales a pu bénéficier du travail de recherche effectuée dans ce cadre. L'objectif de Retro-Color 3D était de trouver une méthodologie permettant de rendre à des objets archéologiques (sculpture peinte ou peinture murale) leurs couleurs d'origine en y intégrant les effets de matières et d'irrégularité qui 
procèdent nécessairement d'un travail artisanal. Le triclinium [7] de la casa di Nettuno ed Anfitrite en a été l'un des cas d'étude.

La restauration numérique des peintures nécessitait de définir les couleurs et leur rapport entre elles le plus justement possible (par mesures colorimétriques) et de trouver comment les reproduire. Les données préalables étaient d'ordre varié : il s'agissait d'une part des éléments de décor in situ et des panneaux détachés conservés au MANN et, d'autre part, d'une documentation connexe composée de photographies, d'ortho-images, de dessins d'archives et enfin de restitutions graphiques produites dans le cadre de l'ANR qui définissent la composition générale du décor.

Les nombreuses photographies collectées présentaient des couleurs très différentes de l'une à l'autre, pour une même zone. Ces différences, liées à l'éclairage, à la diversité des appareils utilisés et de l'ensemble des paramètres qui définissent une prise de vue ne permettaient guère de s'y référer. Il était donc nécessaire d'étudier les couleurs directement sur les peintures originales, mais là encore avec prudence. Il faut noter en effet que les couleurs des peintures in situ ne sont pas toutes fidèles à leur aspect d'origine : certaines ont pu " virer », par exemple les ocres jaunes se transformer en ocres rouges sous l'effet de la chaleur, d'autres se sont patinées ou usées, par exemple par les frottements liés au passage des visiteurs, d'autres encore sont modifiées par la présence de mousses verdâtres. Il en va de même pour les peintures conservées au musée, les panneaux ayant fait l'objet de restaurations à des périodes variées. Certaines zones sont des repeints; des vernis ont également pu être appliqués et s'étant oxydés, ils ont pris une couleur pouvant aller du jaune clair au brun foncé qui modifie fortement le chromatisme de la couche colorée sous-jacente. C'est ainsi que les fonds qui encadrent les niches où se trouvaient des atlantes apparaissent aujourd'hui verdâtre alors que par endroit, le vernis écaillé laisse voir la couleur originale de ce fond plutôt gris bleuté. (fig. 12) Il a donc fallu être particulièrement attentif aux zones choisies lors des mesures de couleurs.

Fig. 12 - Détail d'un des atlantes provenant du triclinium 7 de la Maison de Neptune et Amphitrite à Herculanum, MANN, inv. 8899. L'encadrement apparaît généralement verdâtre sauf là où le vernis de restauration s'est dégradé et où l'on retrouve le gris bleuté original (@ M. Mulliez).

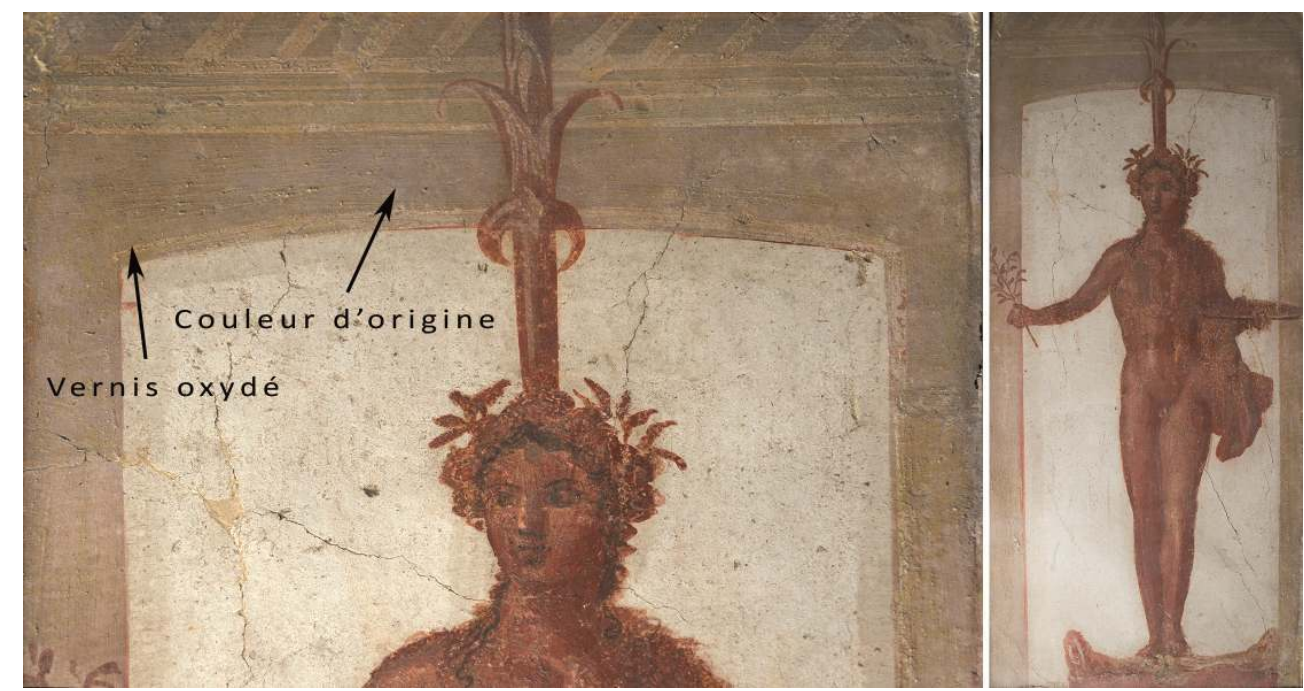




\section{Mesures des couleurs} peintures originales : la spectrocolorimétrie (ou spectrophotométrie) ${ }^{35}$ et la photographie calibrée. Elles ont ensuite été comparées. Le spectrocolorimètre utilisé par l'IRAMATCCRPAA, partenaire du programme, est un Minolta CM-2600d, fonctionnant sous illuminant standard D65 et doté d'une sphère d'intégration qui offre un éclairage homogène. Son domaine spectral est de 360 à $740 \mathrm{~nm}$.

Les paramètres expérimentaux choisis ici étaient : un angle d'observation de $10^{\circ}$ pour une surface analysée de $3 \mathrm{~mm}$ de diamètre, et un temps de mesure de 1,5 secondes avec un intervalle de 3 secondes entre les mesures qui sont ensuite moyennées. Le système permet d'enregistrer divers paramètres comme les spectres de réflectance dont l'allure peut permettre d'identifier la nature des pigments ou les coordonnées chromatiques (en $\mathrm{L}^{*} \mathrm{a}^{*} \mathrm{~b}^{*}$ ) qui, une fois transformées en valeurs RVB peuvent être utilisées dans les logiciels d'infographie afin de reproduire les couleurs mesurées.

Les mesures par photographies calibrées ont été réalisées grâce à l'utilisation d'une charte - ou mire - couleurs, ici est la charte couleurs X.rite Colorchecker, et d'un boîtier Nikon D700. Le principe consiste à photographier la charte couleurs en même temps que le sujet, après avoir fait une balance des blancs; les couleurs de l'image réalisée sont corrigées a posteriori, grâce à la création d'un profil colorimétrique à l'aide du logiciel X.rite Colorchecker fourni avec la charte couleurs.

51 Les deux méthodes ont ensuite été comparées. Sur le panneau du MANN inv. ${ }^{\circ} 8835$ représentant une figure volante sur fond rouge, onze points ont été mesurés à l'aide du spectrocolorimètre. Une fois le profil colorimétrique appliqué à la photographie réalisée, ces mêmes points ont été échantillonnés à l'aide de l'outil «pipette » d'Adobe Photoshop. Ce dernier donne les coordonnées RVB de la zone pointée. Les coordonnées $\mathrm{L}^{*} \mathrm{a}^{*} \mathrm{~b}^{*}$ fournies par le spectrocolorimètre ont été converties en RVB. Puis, afin de les comparer, les résultats ont été appliqués sous la forme d'aplats, fenêtres carrées disposées en deux colonnes, celle des photographies calibrées à gauche, et celle du spectrocolorimètre à droite (fig. 13). 
Fig. 13 - Figure volante provenant du triclinium 7 de la maison de Neptune et Amphitrite à Herculanum, MANN, inv. 883. Comparaison des données colorimétriques obtenue par spectrocolorimétrie et par pointage Photoshop sur une image calibrée.

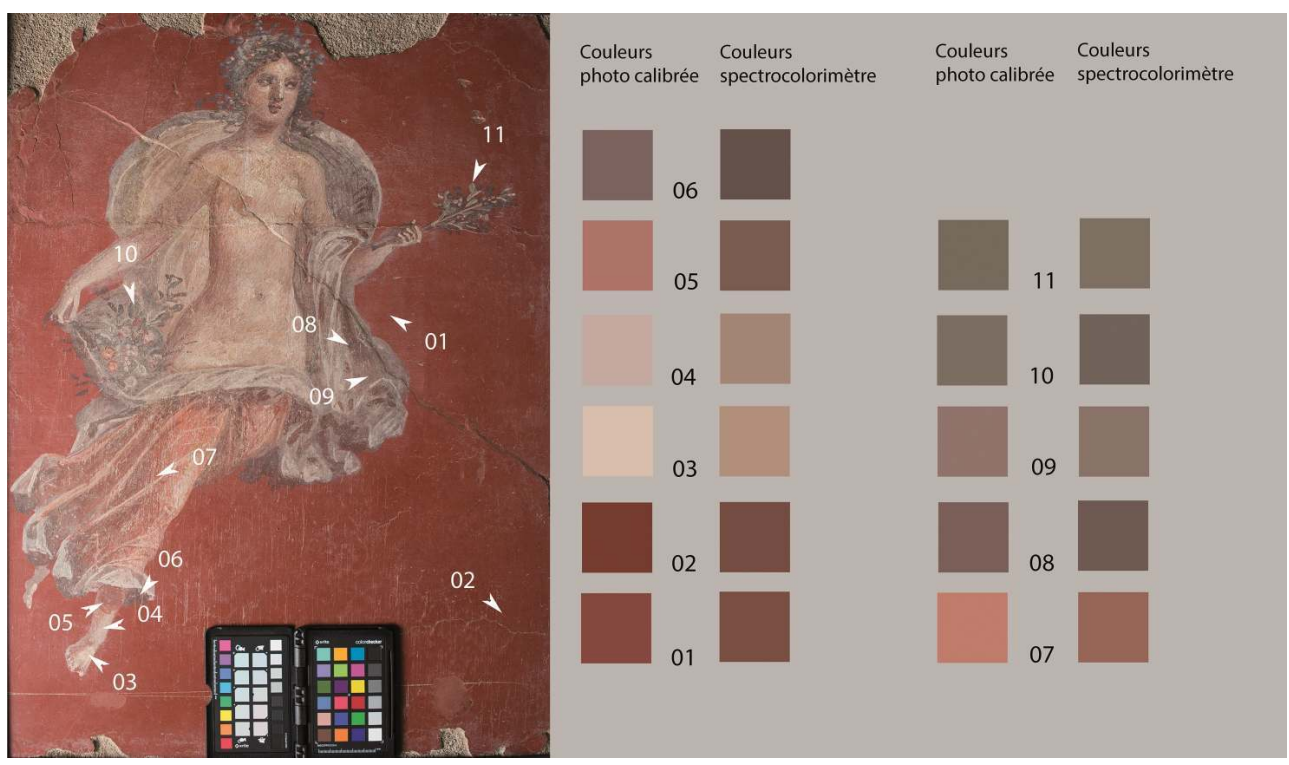

Étant données les conditions de prise de vue dans les réserves du musée où le panneau est situé sur une étagère inclinée et en hauteur, l'éclairage n'a pas pu être uniforme : on voit bien, sur la figure 13, que la partie la plus éclairée est l'angle inférieur gauche de l'image. Dans cette zone, les résultats obtenus par le spectrocolorimètre donnent en effet une couleur légèrement plus sombre que ceux de la photographie (échantillons 03, 04, 05, 06, 07), pour le reste de l'image, les couleurs obtenues dans les aplats comparatifs sont très proches, avec une légère inversion pour l'échantillon 11, le plus éloigné de la source lumineuse.

Cette comparaison des méthodes permet de valider le fait que lorsque l'éclairage est correct - c'est-à-dire homogène, car il peut être rectifié par la balance des blancs - les données obtenues par photographie calibrée sont valables, elles sont confirmées par celles du spectrocolorimètre. Pour la restauration numérique, ce sont les photographies calibrées qui ont été utilisées comme références, du fait que l'on y conserve toutes les nuances alors que le spectrocolorimètre produit une moyenne.

\section{Restauration numérique}

Le terme choisi de « restauration » peut paraitre excessif, si l'on s'en réfère aux usages de la restauration matérielle : en effet, en restauration "classique", la proportion de la surface recréée par rapport à l'original conservé doit rester relativement faible... ici, au contraire, en dehors des figures qui sont virtuellement retouchées mais conservées, les motifs ont été entièrement repris afin de ménager l'unité du décor. Cependant, cette " restauration » étant " numérique », l'œuvre originale n'est en aucun cas mise en danger ou transformée par l'acte de restauration; elle présente également l'avantage de pouvoir passer d'un état à l'autre de l'œuvre, et, en cas d'incertitude, de proposer plusieurs versions. 
55 La composition du décor étant définie au préalable par la restitution graphique, la restauration numérique a consisté à appliquer les bonnes couleurs en reproduisant les effets visuels variés afin de rendre au décor une certaine matérialité. Celle-ci se traduit par des irrégularités, par des couleurs qui, même en aplat, sont en fait parcourues de nuances subtiles, par des effets d'épaisseur sur lesquels la lumière joue. La connaissance de cette matérialité s'acquiert certes par l'observation des peintures originales à la surface desquelles on décèle ici des tracés préparatoires, là une dissymétrie ou une irrégularité dans les motifs, des épaisseurs de couches différentes en fonction des couleurs employées, des traces de pinceaux plus ou moins discernables, etc. Mais l'usure des peintures originales rend aussi cette observation difficile. L'archéologie expérimentale peut alors ici être d'un grand intérêt, à la fois pour retrouver des effets de matières perdues, mais également pour expérimenter les gestes qui permettent de réaliser tel ou tel motif, compréhension qui ne peut qu'être bénéfique pour les reproduire de façon numérique. Le projet a pu bénéficier d'un travail d'archéologie expérimentale déjà réalisés ${ }^{36}$, et ainsi puiser dans la riche documentation qui avait été rassemblée alors, sous la forme de nombreuses photographies d'ensemble, de détails, en lumière rasante (etc.) mais aussi d'un web-documentaire toujours en ligne ${ }^{37}$ pour exploiter un large panel de références visuelles. Les irrégularités de transparence, les taches ou encore les traces de lissage ont ensuite pu être imitées afin de donner à la peinture numérique la « vibration » nécessaire pour la rendre crédible (fig. 14). Celle-ci a été réalisée à l'aide du logiciel Adobe Photoshop dont les outils sont très nombreux et réglables pour la plupart, sur un écran interactif à stylet de 27 pouces. La pression exercée sur la tablette est prise en compte et offre ainsi la possibilité de nuancer un tracé d'une manière très naturelle, procurant une sensation picturale très proche de la réalité. Si la restauration du décor dans son ensemble a en réalité donné lieu à une recréation complète (avec les éléments conservés en sous-main), il en est allé autrement des scènes figurées. Elles n'ont pas été reproduites, mais «restaurées" selon une méthode plus proche de la restauration matérielle : les zones lacunaires ont été comblées, en utilisant soit l'outil «tampon » d' Adobe Photoshop, soit la pipette afin de travailler avec les bonnes couleurs et une série de pinceaux virtuels permettant de repeindre les parties manquantes ou trop altérées. Il a parfois fallu recomposer assez largement les figures: c'est le cas en particulier de la figure volante conservée in situ sur la partie gauche du mur sud et assez largement effacée. C'est en s'inspirant de celle qui devait lui faire pendant obliquement, sur le mur nord, dont le panneau est conservé au MANN (inv. $\mathrm{n}^{\circ}$ 8835) ainsi que d'autres peintures de figures volantes de la même époque, notamment une figure volante de la Casa di Olconio Rufo (VIII, 4.4) à Pompéi, qu'a été recomposée cette figure portant un plateau circulaire (fig.15). Dans le cas où les restes étaient trop ténus pour être recomposés, nous avons opté pour la mise en œuvre d'un fond évoquant par son traitement la présence d'un tableau, sans recréer de scène afin de ne pas sur-interpréter. Les quatre décors restaurés ont ensuite pu être intégrés au modèle 3D de la pièce grâce aux coordonnées conservées des ortho-images (fig. 16).

Fig. 14 - Détail d'une colonne cannelée ornée et surmontée d'un chapiteau provenant du mur ouest du triclinium 7 de la maison de Neptune et Amphitrite à Herculanum et sa « restauration numérique ». On y décèle l'irrégularité volontaire des tracés et des effets de matière comme la variation de transparence et d'opacité liée à la trajectoire du pinceau (au niveau des motifs circulaires blancs : la couleur devient transparente au fur et à mesure du tracé puis au niveau du « levé de pinceau » une accumulation de matière crée un point plus opaque). 


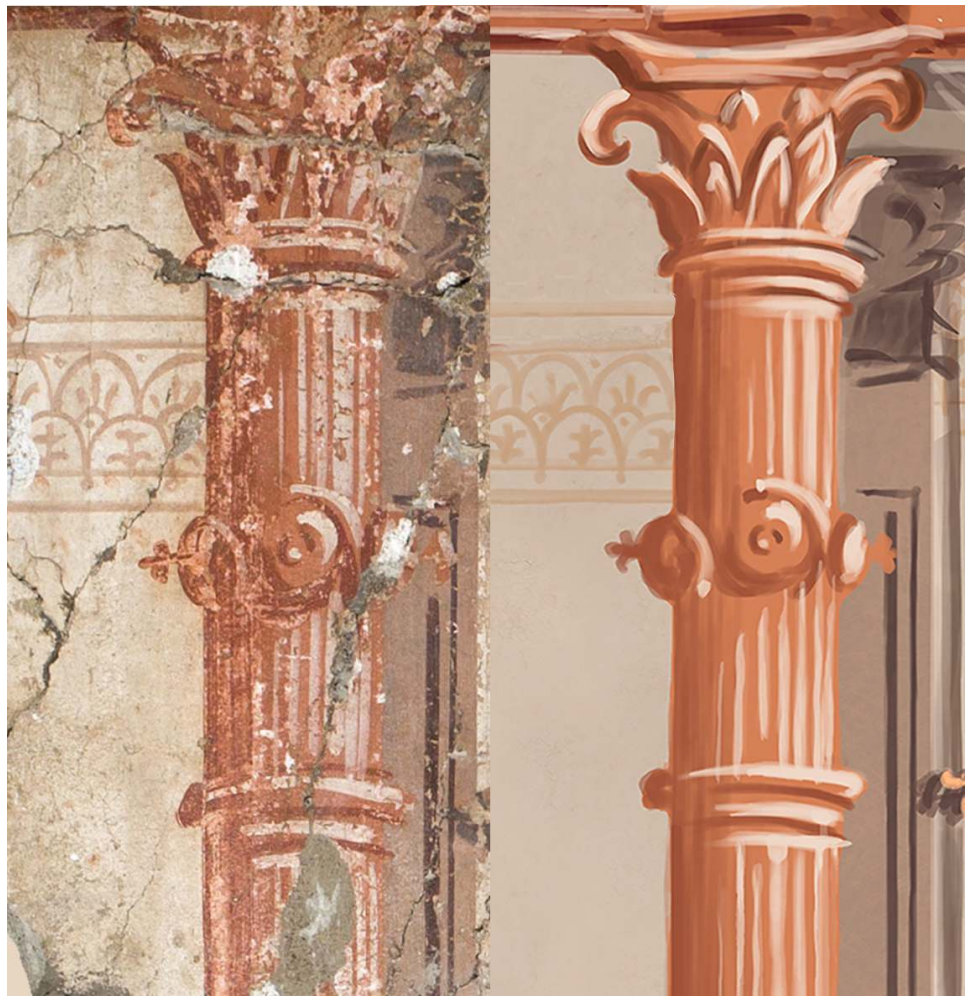

Fig. 15 - Détail de la figure volante de la partie gauche du mur sud du triclinium 7 et sa restauration numérique.

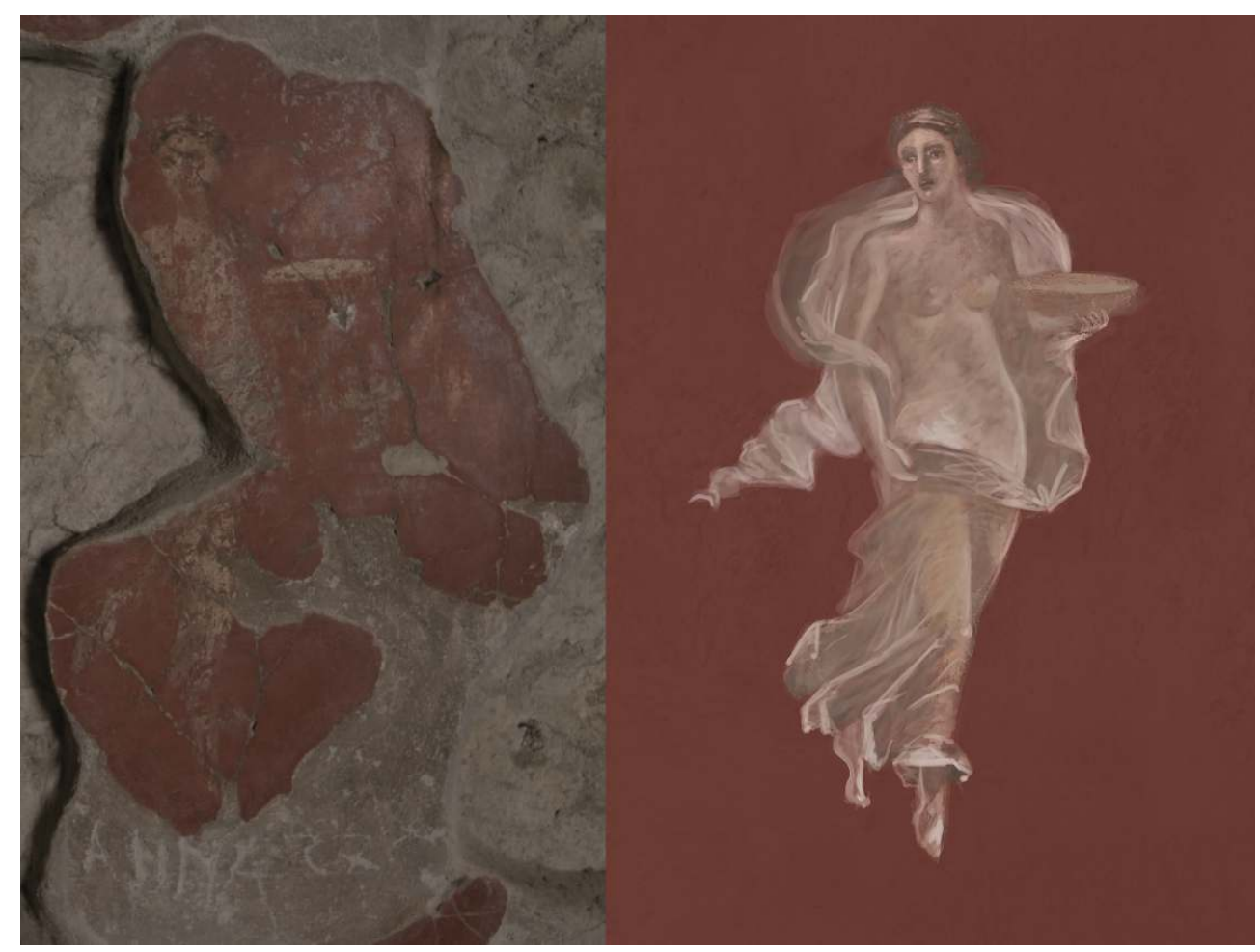


Fig. 16 - Rendu du modèle 3D du triclinium 7 de la maison de Neptune et Amphitrite dont les décors ont été restaurés numériquement. (C) Archéovision/ANR VESUVIA.

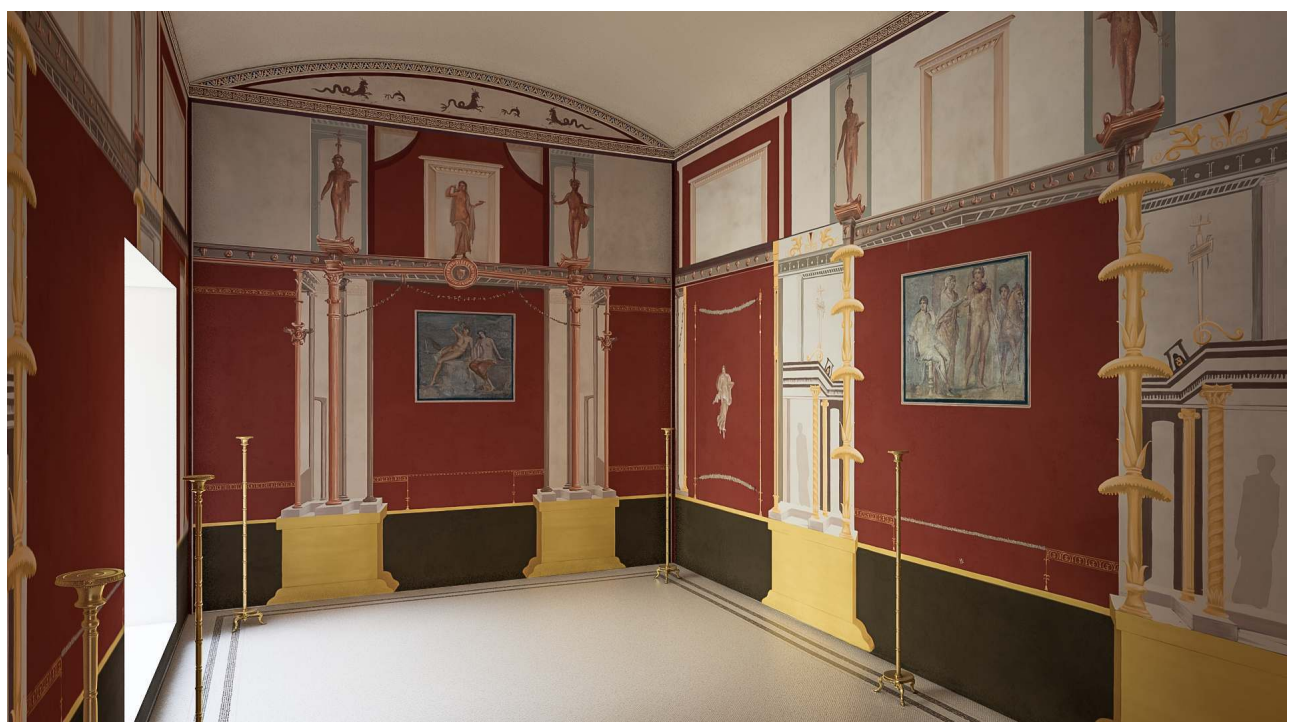

L'expérience archéologique menée par l'équipe du projet VESUVIA, révèle comment le croisement des archives (carnets, photographies, dessins), des relevés archéologiques et de l'analyse in situ, peuvent fusionner dans un modèle numérique soigneusement conçu. Le modèle 3D se transforme, finalement, en chambre d'écho, permettant de rendre intelligible, non seulement aux chercheurs, mais aussi aux néophytes, les résultats d'une longue enquête archéologique, historique et anthropologique.

\section{BIBLIOGRAPHIE}

Aussilloux-Correa- Mulliez 2014 = A. Aussilloux-Correa, M. Mulliez, Re-création d'une fresque antique. Une archéologie expérimentale, dans P. Capus, A. Dardenay, L'empire de la couleur : de Pompéi au sud des Gaules, Toulouse, 2014, p. 18-23.

Baiardi 1755 = O.A. Baiardi, Catalogo degli antichi monumenti dissotterrati dalla discoperta città di Ercolano per ordine della Maestà di Carlo Re delle due Sicilie, e di Gierusalemme, infante di Spagna, duca di Parma, e di Piacenza, gran principe ereditario di Toscana, composto e steso da monsignor Ottavio Antonio Bayardi ...., Naples, 1755.

Boyce 1937= G.K. Boyce, The corpus of the Lararia of Pompei, Rome, 1937 (Memoirs of the American Academy in Rome, 14).

Caruso 2011 = F. Caruso, Melampo e le figlie di Preto. Una lettura del nuovo rilievo di Ercolano, dans RStPomp, 22, 2011, p. 25-35.

Ciotola 2013 = A. Ciotola, Ancora sul rilievo neoattico di Ercolano : una diversa lettura, dans RStPomp, 24, 2013, p. 33-40. 
Dardenay et al. 2016 = A. Dardenay, A. Allroggen-Bedel, H. Eristov, A. Grand-Clément, M.-L. Maraval, C. Marotta, N. Monteix, E. Rosso, Herculanum. Des archives aux restitutions architecturales et décoratives, dans Chronique des activités archéologiques de l'École française de Rome, 2016 (http:// cefr.revues.org/1588, consulté le 9 novembre 2016].

Dardenay et al. 2015 = A. Dardenay, A. Allroggen-Bedel, H. Eristov, M.-L. Maraval, N. Monteix, Habitat et société à Herculanum, dans Chronique des activités archéologiques de l'École française de Rome, 2015.

Esposito 2011 = D. Esposito, Il sistema economico e produttivo della pittura romana. Esempi dall'area vesuviana, dans N. Monteix, N. Tran (éd.), Les savoirs professionnels des gens de métier études sur le monde du travail dans les sociétés urbaines de l'empire romain, Naples, 2011 (Collection du Centre Jean Bérard, 37], p. 65-85.

Esposito 2015 = D. Esposito, $L a$ "Casa dei rilievi dionisiaci ». Osservazioni su una villa urbana ercolanese, dans Aemonitas, IV, 2015, p. 83-142.

Fröhlich $1991=\mathrm{T}$. Fröhlich, Lararien und Fassadenbilder in den Vesuvstädten Untersuchungen zur «volkstümlichen » pompejanischen Malerei, Mayence, 1991 (Mitteilungen des Deutschen Archaeologischen Instituts, Roemische Abteilung. Ergänzungsheft, 32].

Guidobaldi et al. 2014 = F. Guidobaldi, M. Grandi, M.S. Pisapia, R. Balzanetti, A. Bigliati, Mosaici antichi in Italia. Regione prima. Ercolano, Pise-Rome, 2014.

Helbig 1868 = W. Helbig, Wandgemälde der vom Vesuv verschütteten Städte Campaniens, Leipzig, 1868.

Jolivet $2011=\mathrm{V}$. Jolivet, Tristes portiques : sur le plan canonique de la maison étrusque et romaine des origines au principat d'Auguste, Rome, Italie, 2011.

Ling 1997= R. Ling, The Insula of the Menander at Pompeii. Volume I, The structures, Oxford, 1997.

Maiuri 1958 = A. Maiuri, Ercolano. I nuovi scavi, 1927-1958, Rome, 1958.

Monteix $2010=$ N. Monteix, Les lieux de métier. Boutiques et ateliers d'Herculanum, Rome, 2010 (BEFAR , 344).

Nevett 2010= L.C. Nevett, Domestic space in classical antiquity, Cambridge, 2010 (Key themes in ancient history).

Pierattini 2009 = A. Pierattini, Manuale del restauro archeologico di Ercolano : tipi, tecniche costruttive e schede progettuali di indirizzo al restauro dell'edilizia archeologica di Ercolano, Rome, 2009.

Tran $1974=$ T.T. Tran, Catalogue des peintures romaines, Latium et Campanie, du Musée du Louvre, Paris, 1974

Trümper 1998 = M. Trümper, Wohnen in Delos : eine baugeschichtliche Untersuchung zum Wandel der Wohnkultur in hellenistischer Zeit, Rahden, 1998.

Van Andringa 2009 = W. Van Andringa, Quotidien des dieux et des hommes : la vie religieuse dans les cités du Vésuve à l'époque romaine, Rome, 2009 (BEFAR, 337).

Wurmser $2008=\mathrm{H}$. Wurmser, Étude d'architecture domestique : la maison en Grèce à l'époque impériale, Thèse de doctorat, Université Paris-Sorbonne, 2008.

\section{NOTES}

1. Par « évocation ", nous entendons les cas où les fondements scientifiques d'une restitution ne sont pas établis ou rendus accessibles et restent donc invérifiables. 
2. Un bouton pour les escamoter rend la vision plus facile. Pour ceux qui veulent en savoir encore plus, un lien hypertexte renvoie vers nos publications.

3. La maison fut dégagée sous la direction d'Amedeo Maiuri entre novembre 1932 et avril 1934. Des dégâts aux structures, causés lors du creusement des tunnels au XVIII ${ }^{\mathrm{e}} \mathrm{s}$. sont toutefois à regretter.

4. Voir sur la maison grecque et romaine du Haut Empire, les travaux de Nevett 2010, Jolivet 2011, Trümper 1998, Wurmser 2008 , notamment.

5. Guidobaldi et al. 2014, p. 284-285.

6. Les journaux de fouille (GSE 1934, description finale insérée à la fin du mois de juin) donnent les dimensions suivantes : «I travi del compluvio sono di forma rettangolare e misurano m. 0.30 di altezza per m. 0.20 di spessore e sono posti da nord a sud ». Les mesures réalisées sur le mur, non restauré, sont les suivantes : 0,42 m en hauteur, 0,17 m en largeur, mesurées à l'est, en raison de la disparition des tuiles de coffrage dans l'empreinte occidentale.

7. Sans grande spécification, les GSE (1934, description finale insérée à la fin du mois de juin) indiquent les données suivantes pour les chevrons: "I travicelli che sostenevano il tetto misuravano m. 0.10 per 0.9 e la distanza che passa tra l'uno e l'altro è di m. 0.31 ». À l'ouest, la dimension médiane des chevrons est de $0,14 \mathrm{~m}$ en hauteur pour $0,11 \mathrm{~m}$ en largeur, avec un espacement de 0,19 $\mathrm{m}$; au nord, les dimensions médianes sont légèrement supérieures : 0,12 $\mathrm{m}$ en hauteur pour 0,12 m en largeur, avec un espacement de 0,20 m. L'erreur dans les GSE, si elle existe indubitablement, reste contenue.

8. Si les chevrons n'avaient pas été à la même hauteur, il aurait été nécessaire d'insérer les maîtres chevrons dans le pan de mur le plus long - ici les murs septentrional et méridional -, ce génère un noulet à l'intersection des pans de toiture. Pour un exemple d'une telle situation, voir la restitution de la toiture de l'atrium proposée par A. Pierattini (2009, p. 108-109, I ST et Ib ST, p. 111-112) pour la Casa sannitica (V, 1-2). L'auteur privilégie un rapport d'angle de $45^{\circ}$ entre les côtés de l'impluvium et les chevrons de noue pour faciliter la pose des tuiles de noue. Que ce soit dans le cas de la Casa sannitica - dont l'atrium est restauré et donc impossible à étudier - ou d'une manière générale à Herculanum, il ne nous apparaît pas qu'un tel aménagement de charpente soit attesté dans un atrium.

9. GSE 1934: « 7 aprile. Si lavora nella casa $\mathrm{N}^{\circ} 6$ sul $4^{\circ}$ cardine lato est. È stato raccolto sul pavimento dell'ambiente n. 3 una testa di cane di terracotta policromata di bianco. Essa fa parte della decorazione del tetto del compluvio".

10. Le 21 mars 1746, ils se trouvaient dans le triclinium 7, contigu à l'atrium. Cf. Dardenay et al. 2015, § 17-30.

11. Dardenay et al. 2016, § 36 .

12. Dardenay et al. 2016a, annexe (inventaire du mobilier)

13. Boyce 1937 ; Fröhlich 1991.

14. Boyce $1937: \mathrm{n}^{\circ} 67$ (domus Obelli Firmi) ; n¹76 (casa dei Dioscuri) ; n³86 (domus Epidi Sabinus).

15. Ling 1997, pl. 24.

16. Comme le laraire de la Maison dite « de Caecilius Jucundus » $(\mathrm{V}, 1,26)$ à Pompéi.

17. Cf. infra, n. 29.

18. Maiuri 1958, p. 394.

19. Pompéi, I.16.4. Voir Boyce 1937 , notamment Casa del Cenacolo (pl.VIII, 1) ou bien Van Andringa 2009, p.233, 258, 261-264.

20. Dardenay et al. 2016, § 18-21.

21. Cic., Att., 1, 10, 6, 3: "Je te prie en outre de me procurer des bas-reliefs que je puisse enchâsser dans les murs de mon petit atrium, et deux margelles ornées de reliefs » (Traduction L.A. Constans, CUF, 1969)

22. Casa dei rilievi dioniasici, insula I (cf. Caruso 2011, Ciotola 2013, Esposito 2015, ambiente (m), p.107-116). 
23. Tous deux sont partiellement brisés. Inv.150210 (celui signé Alexandros Athenaios) mesure 32 $\mathrm{cm}$ (ht) par $39 \mathrm{~cm}$ (lg), inv. 150211 mesure $35 \mathrm{~cm}$ ht par $47 \mathrm{~cm}$ (lg). Pour restituer les dimensions initiales des pinakes il est possible de s'appuyer sur un autre exemplaire mis au jour à Herculanum au XVIII siècle et conservé sous l'inv. nº 9562 (les "Joueuses d'Osselet», signé Alexandros Athenaios) quasiment intact qui mesure $49 \mathrm{~cm}(\mathrm{ht})$ par $42 \mathrm{~cm}$ (lg).

24. Maiuri 1958, p. 397.

25. CIL IV, 10 480, cf. Monteix 2010, p. 329-330 ; Dardenay et al. 2016, § 36, 45.

26. Voir notamment l'exemple de la Casa dei pittori al lavoro (IX 12,9) à Pompéi (Esposito 2011, p. 69-75).

27. Maiuri 1958, fig. 332 p. 396 ; Alinari 43124.

28. GSE 1934 ; Maiuri 1958, 397.

29. Rapport d'étude par Delphine Burlot, août 2010. Le fragment donné au roi de France en 1825 avait subi de nombreux repeints. Voir Tran 1974

30. Baiardi 1755, 29.

31. https://digitalmontagny.inha.fr/fr/node/667.

32. RPGR 196.8 ; RMB I, 4 ; Helbig 1868 (1364).

33. Helbig 1868 (1365) note que le tableau a été prélevé, mais n'en trouve pas trace au musée ; l'identification avec le panneau du musée (Helbig 1363) est due à I. Bragantini (EAA-PPM IV, 1993, 449 et fig. 11 p. 455) qui, à la suite de Warsher Codex pense que Helbig a décrit deux fois le même tableau; cependant des différences substantielles existent entre les deux descriptions; mais surtout une certaine imprécision règne sur les provenances : la notice du Real Museo Borbonico, vol. I, pl. IV (1824) donne Helbig 1364 comme provenant de la maison des Dioscures.

34. Pour I. Bragantini, la phase de quatrième style de cette vaste maison ne se signale d'ailleurs pas par sa qualité: «schemi ricchi di effetto ma non di particolare impegno » (PPM IV, 1993, 449) ; seul le tablinum comportait des tableaux.

35. Ces mesures ont été réalisées en collaboration avec l'IRAMAT-CRPAA (Institut de Recherche sur les ArchéoMATériaux - Centre de Recherche en Physique Appliquée à l'Archéologie, UMR CNRS 5060) : nous tenons à remercier Rémy Chapoulie, Floréal Daniel et Aurélie Mounier pour le prêt du spectrocolorimètre lors d'une mission à Herculanum en avril 2016 et pour les analyses réalisées ensemble consécutivement.

36. Dans le cadre de l'exposition L'Empire de la couleur, de Pompéi au Sud des Gaules qui s'est tenu du 15 novembre 2014 au 22 mars 2015 au Musée Saint-Raymond, musée des Antiques de Toulouse sous le commissariat scientifique de Pascal Capus et Alexandra Dardenay.

37. Aussilloux-Correa - Mulliez 2014; http://www.tectoria-romana.com .

\section{AUTEURS}

\section{ALEXANDRA DARDENAY}

Université Toulouse II Jean Jaurès, UMR 5608 « TRACES » (CNRS / Université de

Toulouse II) - adardenay[at]yahoo.fr

\section{HÉLÈNE ERISTOV}

UMR 8546 « AOROC » (CNRS / ENS) - helene.eristov[at]ens.fr 


\section{ADELINE GRAND-CLÉMENT}

Université Toulouse Jean Jaurès, PLH-ERASME - adelinegc[at]yahoo.fr

\section{MARIE-LAURE MARAVAL}

Université Toulouse II Le Mirail, DAR (Direction Appui Recherche), UMR 5608 « TRACES » (CNRS / Université de Toulouse II) - mmaraval[at]univ-tlse2.fr

\section{NICOLAS MONTEIX}

Université de Rouen, GRHis (EA 3831) - nicolas.monteix[at]univ-rouen.fr

\section{PASCAL MORA}

UMS 3657 SHS 3D « Archéovision » - pascal.mora[at]u-bordeaux3.fr

\section{MAUD MULLIEZ}

UMR 7041 « ArScAn », UMS 3657 SHS 3D « Archéovision » - maud[at]maudmulliez.comFR 